\title{
Lemniscal recurrent and transcortical influences on cuneate neurons
}

\author{
A. Canedo, J. Mariño, J. Aguilar
}

\begin{abstract}
Intracellular recordings were obtained from cuneate neurons of chloralose-anesthetized, paralysed cats to study the synaptic responses induced by electrical stimulation of the contralateral medial lemniscus. From a total of 178 cells sampled, 109 were antidromically fired from the medial lemniscus, 82 of which showed spontaneous bursting activity. In contrast, the great majority (58/69) of the non-lemniscal neurons presented spontaneous single spike activity. Medial lemniscus stimulation induced recurrent excitation and inhibition on cuneolemniscal and nonlemniscal cells. Some non-lemniscal neurons were activated by somatosensory cortex and inhibited by motor cortex stimulation. Some other non-lemniscal cells that did not respond to medial lemniscus stimulation in control conditions were transcortically affected by stimulating the medial lemniscus after inducing paroxysmal activity in the sensorimotor cortex. These findings indicate that different sites in the sensorimotor cortex can differentially influence the sensory transmission through the cuneate, and that the distinct available corticocuneate routes are selected within the cerebral cortex. From a total of 92 cells tested, the initial effect induced by low-frequency stimulation of the sensorimotor cortex was inhibition on most of the cuneolemniscal neurons (32/52) and excitation on the majority of the non-lemniscal cells (25/40). The fact that a substantial proportion of cuneolemniscal and non-lemniscal cells was excited and inhibited, respectively, suggests that the cerebral cortex may potentiate certain inputs by exciting and disinhibiting selected groups of cuneolemniscal cells. Finally, evidence is presented demonstrating that the tendency of the cuneolemniscal neurons to fire in high-frequency spike bursts is due to different mechanisms, including excitatory synaptic potentials, recurrent activation through lemniscal axonal collaterals, and via the lemniscothalamo-cortico-cuneate loop.
\end{abstract}

A corticocuneate network circuit to explain the results is proposed.

Keywords: somatosensory system; burst firing; recurrent collaterals; corticocuneate input; cat

Abbreviations: CL, cuneolemniscal neuron; CN, cuneate nucleus; DCN, dorsal column nuclei; ECoG, electrocorticogram; EPSP, excitatory postsynaptic potential; GABA, $\gamma$-aminobutyric acid; IPSP, inhibitory postsynaptic potential; ML, medial lemniscus; nCL, non-cuneolemniscal neuron; VPL, ventroposterolateral thalamic nucleus

The somesthetic afferent fibers to the cuneate nucleus synapse with intranuclear neurons and projection cells. The great majority of neurons in the middle cuneate region $(0-4 \mathrm{~mm}$ caudal to the obex in the cat) responds either to movement of hairs, or to touch or pressure applied to the skin. Some respond to movement of joints or to stimulation of deep receptors. Most of the input to the dorsal column nuclei (DCN: cuneate and gracilis), especially to their middle regions, is from primary afferent fibers carrying low-threshold, unimodal information from the $\operatorname{skin}^{18}$ and conserve the somatotopic organization seen in dorsal column fibers at cervical levels. ${ }^{8}$ and 39 . The primary afferents terminate mostly within the clusters zone $^{32}$ or cellular bricks ${ }^{8}$ where lemniscal-projecting neurons predominate. ${ }^{21 ., 27 .}$ and 32 . The local neurons of this region have larger and more proximal cutaneous receptive fields than the lemniscal-projecting cells. ${ }^{13}$ Neurons located rostrally and caudally to the clusters region have, on average, larger peripheral receptive fields. ${ }^{24 . ~ a n d ~} 26$.

It is well known that the cuneate neurons responding to cutaneous stimulation tend to discharge in high-frequency bursts of two to five spikes. and 43. increasing their efficacy on target neurons. This tendency to discharge in bursts can be partially explained by giant postsynaptic excitatory potentials (EPSPs) monosynaptically induced by activation of primary afferent fibers. ${ }^{3 \text { and }}$ 7. To further augment their postsynaptic impact, the ensembles of cuneolemniscal (CL) neurons receiving input from a particular peripheral receptive field might fire synchronously. The divergence of primary afferent fibers ascending in the dorsal columns provides a synchronizing mechanism that would depend on (i) the number of afferents activated in response to a peripheral stimulus and (ii) their conduction velocities, as well as their degree of divergence and their synaptic efficacy. This divergence appears, however, limited 
given the small receptive fields of the CL neurons. Additional mechanisms to synchronize CL neurons may be necessary to drive thalamocortical cells of the ventroposterolateral (VPL) nucleus. The activation of CL neurons by intranuclear recurrent collateral branches of CL axons and via the lemniscal-thalamocortico-cuneate loop might constitute two linked synchronizing mechanisms.

The CL fibers emit recurrent collaterals before entering the medial lemniscus (ML) ${ }^{10 .}$ and 22. and ML stimulation induces synaptic responses in the DCN. These transynaptic responses have been ascribed to recurrent intranuclear inhibition or excitation, 2., 23., 24., 25., 26. and 27. to cortical reflexes induced by the lemniscal ascending activity affecting the nucleus by a transcortical route, ${ }^{51}$ and to dorsal column reflexes generated in primary afferent terminals by presynaptic depolarization. ${ }^{5 .}$, 30 and 50 . Thus, electrical volleys applied to the ML should induce a double discharge in the DCN, the first being produced through recurrent collaterals of activated lemniscal axons and the second by the activation of the thalamo-corticoDCN loop. This would imply that the corticofugal discharges excite lemniscal neurons, 1., 15., 24., 25., 29. and 46. which is in disagreement with the model proposed by Andersen et al. ${ }^{6 .}$ and 7 . for the synaptic organization of the cuneate nucleus. These authors suggested that the cerebral cortical fibers drive interneurons mediating inhibition on to CL cells. By this model, the primary afferent fibers synapsing with CL neurons would be subjected to presynaptic inhibition mediated by interneurons activated by corticofugal input. In agreement with this idea, it was recently reported that cortical stimulation activated the cuneate nonlemniscal (nCL) and inhibited the CL neurons. ${ }^{13}$ These data were, however, obtained by stimulating the primary motor cortex (area 4 or MI) and, thus, the possibility exists that the cortical excitatory effects on CL cells reported by others might be particularly seen when stimulating the primary somatosensory cortex (SI), as has been suggested based on extracellular recordings from the cuneate (cat ${ }^{16}$ and 33 , rat ${ }^{47}$ ) and gracile (cat ${ }^{16 .}$, 24. and 25 .) nuclei.

According to the above, this work was aimed to study the synaptic responses induced by ML electrical stimulation on intracellularly recorded $\mathrm{nCL}$ and CL neurons. Electrical stimulation and inactivation of the sensorimotor cortex allowed a distinction to be made between recurrent and transcortical responses induced by ML volleys. This also permitted us to test the Andersen et al. ${ }^{6 \text { and } 7 \text {. model. }}$

Finally, recordings from cuneate cells with resting membrane potentials below $-50 \mathrm{mV}$ permitted us to reveal spontaneous giant EPSPs leading to high-frequency bursting activity.

Preliminary results have been reported in abstract form. ${ }^{12}$

\section{Experimental procedures}

All experiments conformed to Spanish guidelines (BOE67/1988) and European Communities Council Directive (86/609/EEC) and all efforts were made to minimize the number of animals used.

Data were obtained from a total of 30 cats of either sex (2.5-4.2 kg), anesthetized ( $\alpha$-chloralose, 60 $\mathrm{mg} / \mathrm{kg}$, i.p.), paralysed (Pavulon $1 \mathrm{mg} / \mathrm{kg} / \mathrm{h}$, i.v.) and artificially respired. The methods have already been described in full. ${ }^{37}$ In short, a bipolar stimulating electrode served to stimulate the ML, and a set of six bipolar stimulating electrodes was mounted in a tower and lowered to $1-1.5 \mathrm{~mm}$ deep in the pericruciate cortex to stimulate corticocuneate neurons. The cortical electrodes were disposed in two rows aligned mediolaterally in the anterior and posterior gyrus sigmoideus so that primary motor and primary somatosensory cortices could be stimulated (see Fig. 8A). In three animals, the cortex was inactivated for several hours by placing a piece of cotton soaked with the $\gamma$-aminobutyric acid (GABA) agonist muscimol $(0.5 \mathrm{mg}$ in $0.5 \mathrm{ml}$ of saline) over the sensorimotor cortical surface. This served to block the corticocuneate transmission to suppress the transcortical responses to lemniscal stimulation. A bipolar concentric electrode with the inner lead having a resistance of about $50 \mathrm{~K} \Omega$ and separated by $500 \mu \mathrm{m}$ from the ring was routinely placed in the lateral tip of the cruciate sulcus at a depth of $1 \mathrm{~mm}$ to record the electrocorticogram (ECoG). Sharp electrodes filled with $2.5 \mathrm{M}$ of $\mathrm{K}^{+}$acetate and with resistances measured in vivo of 30-50 M $\Omega$ were used to record intracellularly from cuneate neurons. All CL cells satisfied the collision test with spontaneous action potentials ( Figs 2C1, 4A, 6B1). Manipulation of the peripheral receptive fields in the distal ipsilateral forelimb and reconstruction of the electrode tracks confirmed that the recordings were obtained from the middle cuneate nucleus. 


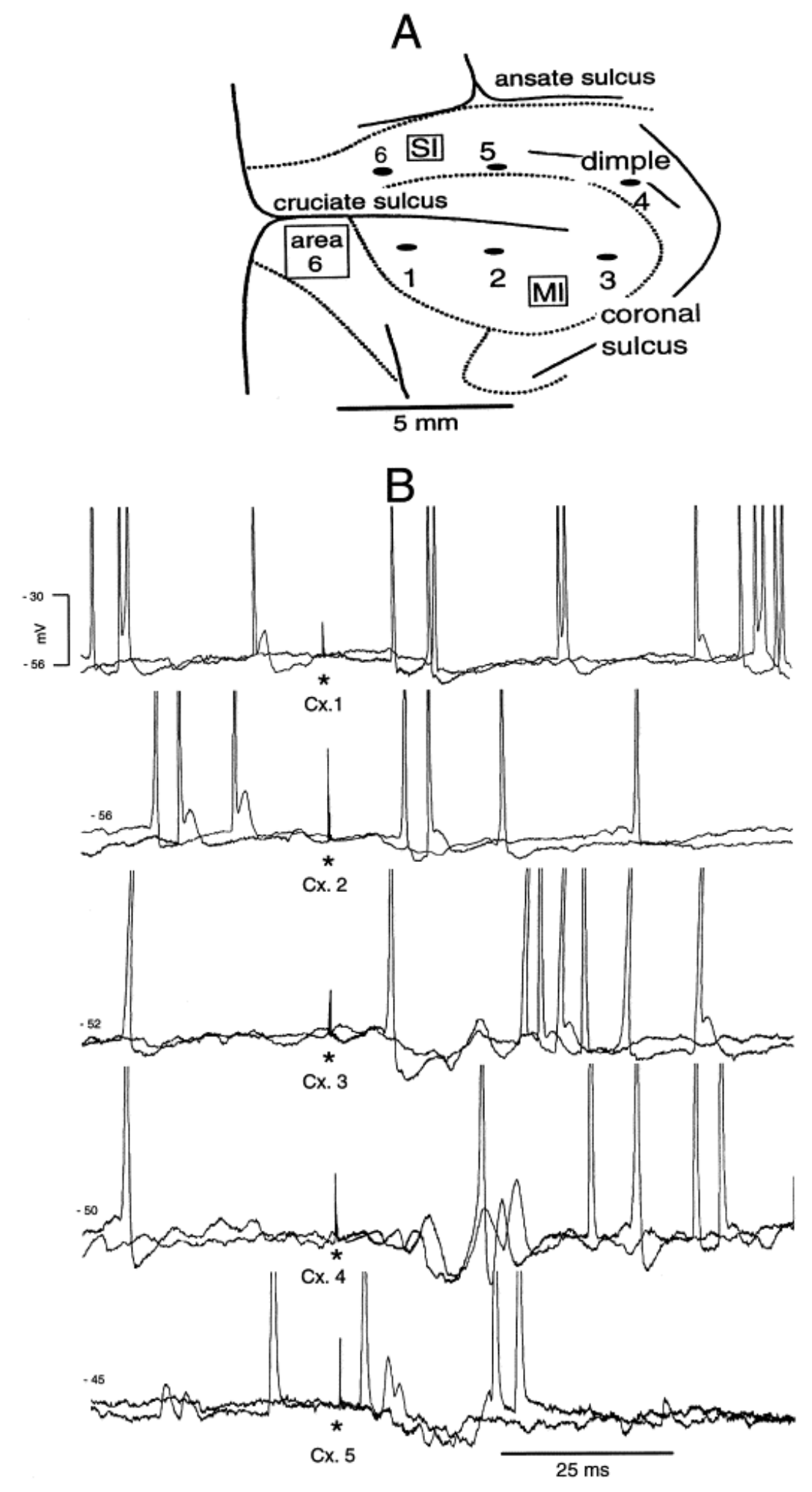

Fig. 8. Sensorimotor cortical stimulation at distinct sites differentially affected CL cells. (A) Diagram showing the relative placement of six bipolar stimulating electrodes in the sensorimotor cortex. (B) Same cell as in Fig. 7. Medial and intermediate precruciate motor cortex stimulation (sites 1 and 2 in the diagram shown in A) induced inhibitory effects (two upper panels in B) while forelimb motor cortex (site 3 ) and primary somatosensory cortex (sites 4 and 5) evoked excitatory-inhibitory sequences (last three panels). Stimulus artifacts are marked by asterisks. The spikes were truncated. 


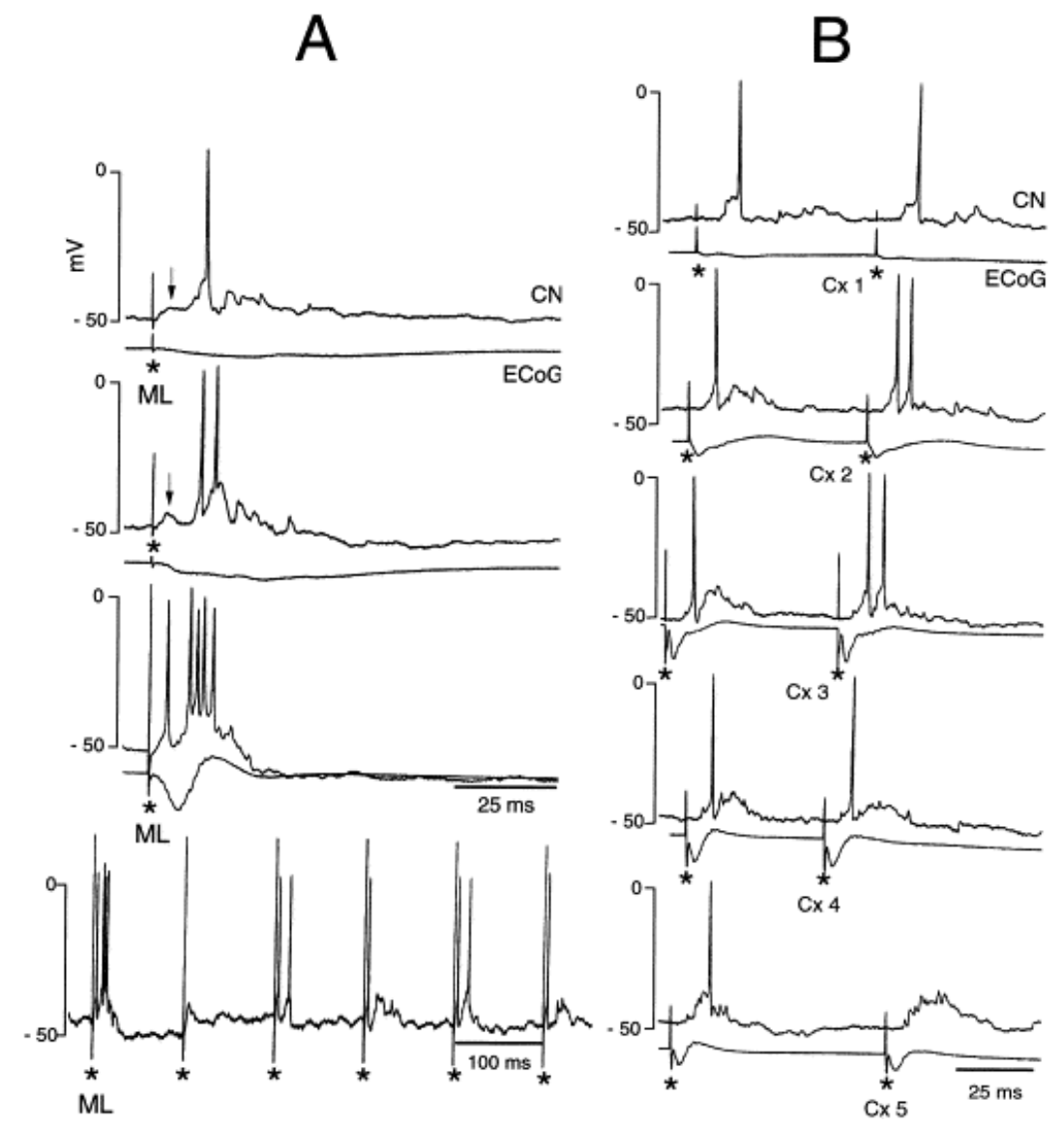

Fig. 2. Composite recurrent and transcortical excitatory responses on a nCL cell. Increasing the ML stimulation intensity (A, first three panels) generated recurrent EPSPs (signaled by arrows) leading to a short-latency ( $\sim 3.5 \mathrm{~ms})$ full spike (third panel), followed by a transcortical depolarization generating bursting discharges with the number of action potentials in each burst increasing with stimulating strength. Suprathreshold ML stimulation at $10 \mathrm{~Hz}$ (A, lower row) greatly reduced the transcortical responses. The excitatory responses induced by stimulating different sites within the sensorimotor cortex (see Fig. 8A) are shown in B. The first record of each pair is the intracellular cuneate recording $(\mathrm{CN})$ and the second corresponds to the electrocorticogram $(\mathrm{ECoG})$. Stimulus artifacts are marked by asterisks.

\section{Results}

\subsection{General}

The study is based on a total of 178 cells spontaneously active with resting membrane potentials from -45 to $-75 \mathrm{mV}$ and that could be maintained in good conditions during the different experimental tests performed. A total of 109 of these cells was antidromically identified as CL neurons, while the remaining 69 failed to be antidromically fired from the contralateral ML and thus are considered as nCL cells or putative interneurons. The CL neurons tended to generate bursts of two to five spikes (82/109) whether or not intermingled with single spikes. The bursting activity was particularly evident at membrane potentials negative to $-50 \mathrm{mV}$ while at more depolarized values the bursts were usually replaced by single spike activity. In contrast, most of the nCL neurons (58/69) presented single spike activity. Ninety-two of the cells $(52 \mathrm{CL}$ and $40 \mathrm{nCL})$ were obtained from experiments previously reported ${ }^{37}$ and analysed in relation to the synaptic effects induced by low-frequency $(1-10 \mathrm{~Hz})$ stimulation of the sensorimotor cortex.

To study recurrent responses, the ML was routinely stimulated at low and high repetitive rates. Once a presumed recurrent effect was detected at 1-2 $\mathrm{Hz}$ stimulation rates, higher stimulating frequencies of up to $200 \mathrm{~Hz}$ were subsequently applied to obtain an indication of the number of synapses involved. 


\subsection{Recurrent excitatory responses}

ML stimulation induced EPSPs, presumably generated by recurrent collateral branches from CL neurons, on 32 out of $55 \mathrm{CL}$ cells and on eight out of $29 \mathrm{nCL}$ neurons tested. The mean latency of these synaptic responses was $2 \pm 0.5 \mathrm{~ms}$ (mean $\pm \mathrm{SD}$ ). To uncover the presumed recurrent EPSPs, positive current was injected through the recording electrode since sustained membrane hyperpolarization induced rhythmic oscillations of the membrane potential that made it difficult to study the synaptic responses. Membrane depolarization close to $-20 \mathrm{mV}$ was necessary to uncover the EPSPs responsible of the presumed recurrent spikes. These short-latency spikes could be blocked in five CL cells in which they had precisely the same threshold as the antidromic responses. This procedure was not necessary either for the CL cells showing different thresholds for both responses or for the nCL neurons since, in both cases, the recurrent effects could be distinguished by varying the intensity of ML stimulation.

2.2.1. Cuneolemniscal cells. One to three transynaptic spikes followed the antidromic action potentials induced by ML stimulation. These presumed recurrent excitations followed $50-200 \mathrm{~Hz}$ iterative stimulation indicating their probable monosynaptic nature, and were mostly seen in spontaneously bursting CL cells (24/32). The examples shown in Fig. 1 illustrate a double spiking (A) and a tonically discharging (B) CL neuron showing recurrent responses linked to (A) and independent of (B) a preceding spike. The spontaneous spike doublets generated by the cell shown in Fig. 1A emerged from depolarizations resembling synaptic potentials. A train of $320 \mathrm{~ms}$ duration applied to the ML at $200 \mathrm{~Hz}$ induced antidromic action potentials and a sustained hyperpolarization (A2). The first stimulus of the lemniscal tetanus induced an antidromic response followed by a transynaptic action potential that persistently failed during the remainder of the train. The second spike in the doublet arose from a delayed depolarization (marked by black dots). Note also that the fast afterhyperpolarization clearly visible at the beginning of the train decreased as the membrane hyperpolarized, thus indicating its voltage dependence. The presumed recurrent spike was blocked by membrane depolarization, uncovering the underlying EPSP (marked by an arrow in the lower row of Fig. 1A3). The recurrent response of the neuron shown in Fig. 1B was not linked to a previous spike and had a lower threshold than the antidromic response. It appeared at about $2 \mathrm{~ms}$ latency (Fig. 1B1, signalled by an arrow in the lower panel) and followed ML stimulating frequencies up to $100 \mathrm{~Hz}$. ML stimulation at $1 \mathrm{~Hz}$ also induced a late hyperpolarization leading, in most cases, to rebound action potentials (Fig. 1B1) which disappeared at stimulating frequencies of $10-50 \mathrm{~Hz}$ (Fig. 1B2), suggesting its multisynaptic nature. In summary, the presumed ML recurrent responses may or may not be linked to preceding spikes, suggesting that they could be induced by recurrent axons from the same and other CL neurons, respectively. 


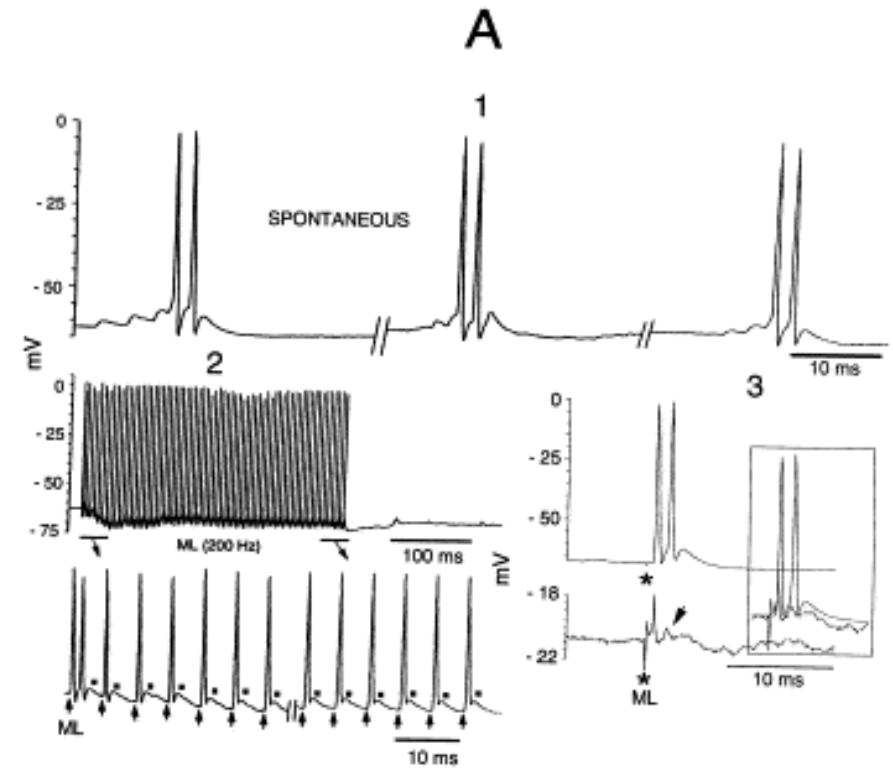

B

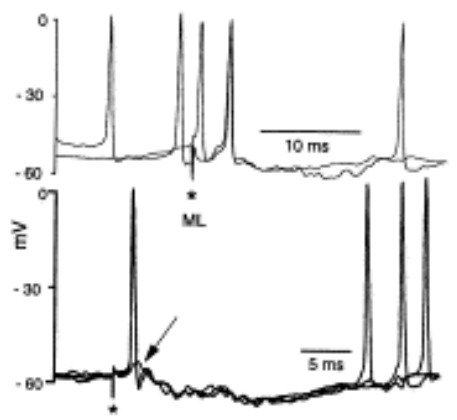

M.

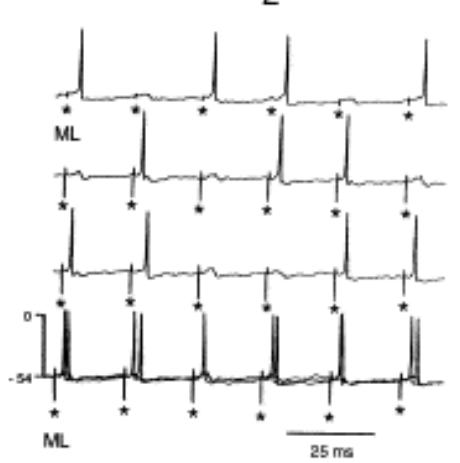

Fig. 1. Lemniscal recurrent excitation. (A) A spontaneous bursting CL cell (A1) with recurrent excitation linked to a previous spike following $200 \mathrm{~Hz}$ medial lemniscal (ML) stimulation (A2, the portions marked by horizontal bars are expanded below; the black squares signal the presumed recurrent depolarization). Low-frequency ML stimulation induced an antidromic spike followed by recurrent responses (A3, upper row; B2). The recurrent postsynaptic potential was unmasked upon membrane depolarization (A3, lower row. Both rows are superimposed in the inset). (B) A spontaneous tonic CL cell with recurrent excitation not linked to a previous spike. Recurrent EPSPs followed the antidromic spikes (B1, upper: two superimposed sweeps in one of which collision between an orthodromic and one antidromic spike occurred) which also appeared at subthreshold stimulation for antidromic activation (B1, lower: the arrow points to a recurrent subthreshold EPSP). Subthreshold ML stimulation for antidromic activation at $50 \mathrm{~Hz}$ induced sequences of excitatory-inhibitory responses with full spikes eventually crowning the EPSPs (B2, the first three rows superimposed in the lower panel) and suppressed the late inhibitory response generated by stimulating at $1 \mathrm{~Hz}$ (B1). Stimulus artifacts are marked by asterisks and arrows (A2).

2.2.2. Non-cuneolemniscal cells. A total of eight $\mathrm{nCL}$ neurons (seven single spiking, one bursting) showed ML-recurrent excitatory effects. The presumed recurrent EPSPs followed 50-100 Hz ML iterative stimulation. The ML stimuli also activated these $\mathrm{nCL}$ cells through the cerebral cortex. The example shown in Fig. 2 serves to illustrate the behavior of these neurons. Increasing strengths of ML stimulation generated compound EPSPs crowned by one or more spikes at low intensity (Fig. 2A, two upper intracellular records) and sequences of EPSPs-IPSPs at a higher intensity, with the excitatory preceding the inhibitory effect (Fig. 2A, third intracellular trace). Note that the excitatory responses were constituted by a rapid EPSP (signaled by arrows), presumably due to recurrent excitation, and by latter compound EPSPs leading to repetitive activity, presumable due to cortical reflex responses since the cell was also activated by sensorimotor cortical stimulation at a shorter latency (Fig. 2B) and similar late responses were abolished by sensorimotor cortical inactivation (e.g. Fig. 6B). 

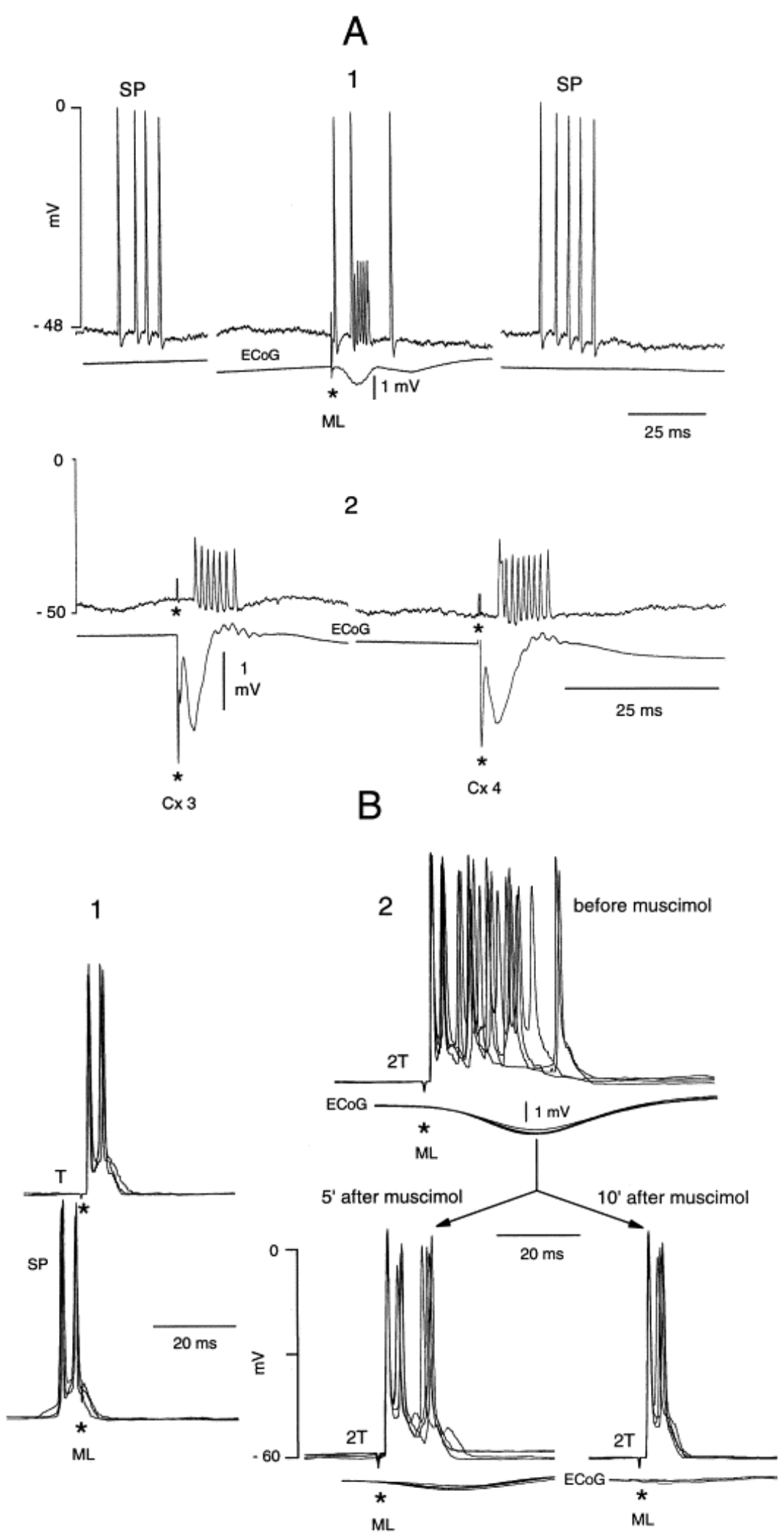

Fig. 6. Transcortical excitation. Two different CL neurons (A, B). (A) ML stimulation generated a transcortical response following the antidromic spike (A1, middle). Stimulation of primary forelimb motor $(\mathrm{Cx} 3)$ and somatosensory $(\mathrm{Cx} 4)$ cortices induced bursts of incomplete spikes (A2). (B) the CL cell illustrated (antidromic identification in B1) generated recurrent responses linked to the presence of a previous spike (B1, SP=spontaneous). Increasing the ML stimulating intensity elicited transcortical responses (B2, upper) that gradually disappeared after cortical inactivation (B2, lower). The lower record of each pair (A2 and B2) is the electrocorticogram (ECoG). Stimulus artifacts are marked by asterisks. 


\subsection{Recurrent inhibitory responses}

2.3.1. Cuneolemniscal neurons. ML stimulation induced IPSPs on 14/55 CL cells (11 bursting, three single spiking) that lagged the ML stimuli by $2.5-4.5 \mathrm{~ms}$ and by $11.5 \pm 2.5 \mathrm{~ms}$. These double effects were seen in the same neurons (7/14) as well as in different cells (two showed short-latency inhibition and five long-latency inhibition). These responses were probably induced by recurrent ML collaterals (the first inhibition) and through the cerebral cortex (the late inhibition). The short-latency inhibitory responses followed ML stimulation at rates of $15-30 \mathrm{~Hz}$ while the late inhibition followed ML stimulating rates of 5-10 Hz. Figure 3 shows an example illustrating the short latency inhibition induced on a double spiking CL neuron. The cell generated a presumed recurrent IPSP which increased in amplitude with increasing ML stimulation intensity (Fig. 3B). The late inhibitory responses are separately described in the section "Transcortical inhibitory responses".

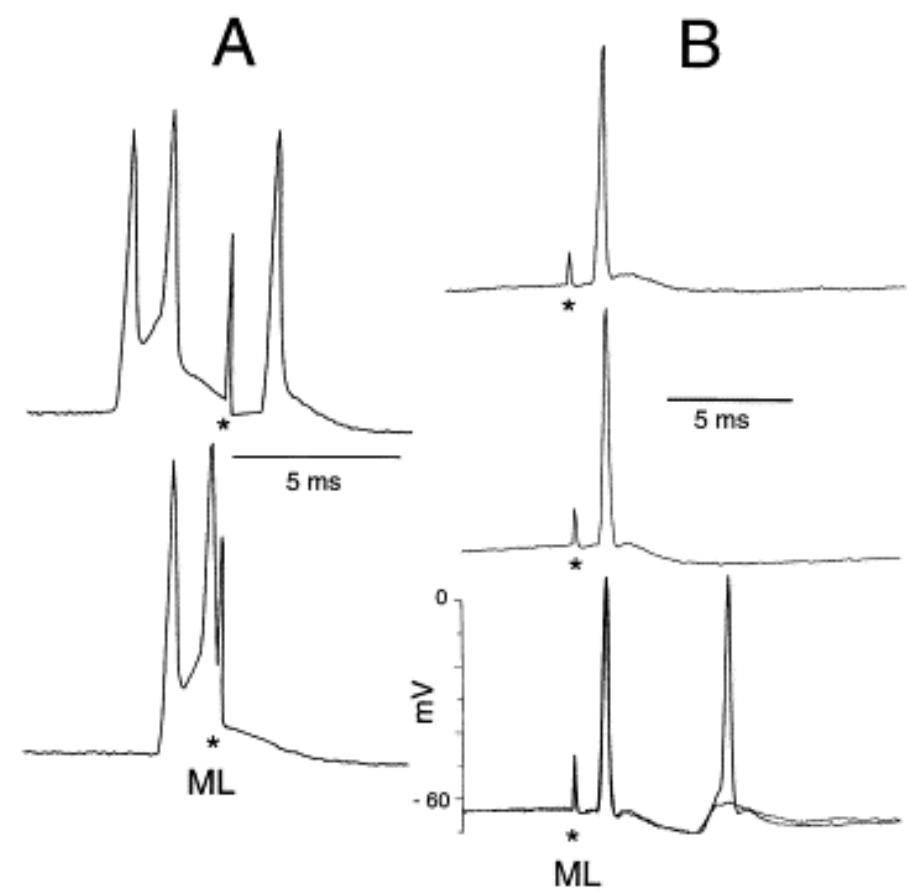

Fig. 3. Recurrent inhibition on a CL neuron. The antidromic identification is illustrated in A (collision in the second row). ML stimulation generated excitatory-inhibitory sequences following the antidromic spike; the inhibition increased gradually with increasing stimulation intensity (B, first to second row: average of 10 sweeps each) until leading to a rebound excitation (B, third row: two superimposed sweeps). Stimulus artifacts are marked by asterisks. 
2.3.2. Non-cuneolemniscal cells. A total of $7 / 29 \mathrm{nCL}$ neurons were inhibited by both $\mathrm{ML}$ and somatosensory cortex (SSCx) stimulation at a latency of $2.5 \pm 0.6 \mathrm{~ms}$ (range 1.3-4 ms; frequency following to ML stimulation: $15-30 \mathrm{~Hz}$ ) and of $6.8 \pm 1.5 \mathrm{~ms}$ (range 5-8.5 ms; frequency following to ML stimulation: 5-10 Hz), respectively. The sample records of Fig. 4 exemplify the behavior of these cells. The neuron illustrated in Fig. 4A generated inhibitory responses to ML (latency about $1.3 \mathrm{~ms}$ ) and to forelimb motor cortex (latency about $5 \mathrm{~ms}$ ) stimulation (Fig. 4A). Note that suprathreshold ML stimuli generated a compound IPSP which could be separated into two components by gradually increasing the intensity of stimulation (Fig. 4B). The second IPSP had a latency of about $6 \mathrm{~ms}$ which indicates that it hardly could be induced through a transcortical route since direct cortical stimulation induced a similar response at a latency of $5 \mathrm{~ms}$. The compound IPSPs with latencies not compatible with being produced through the cerebral cortex were probably generated by recurrent branches of CL axons.

A
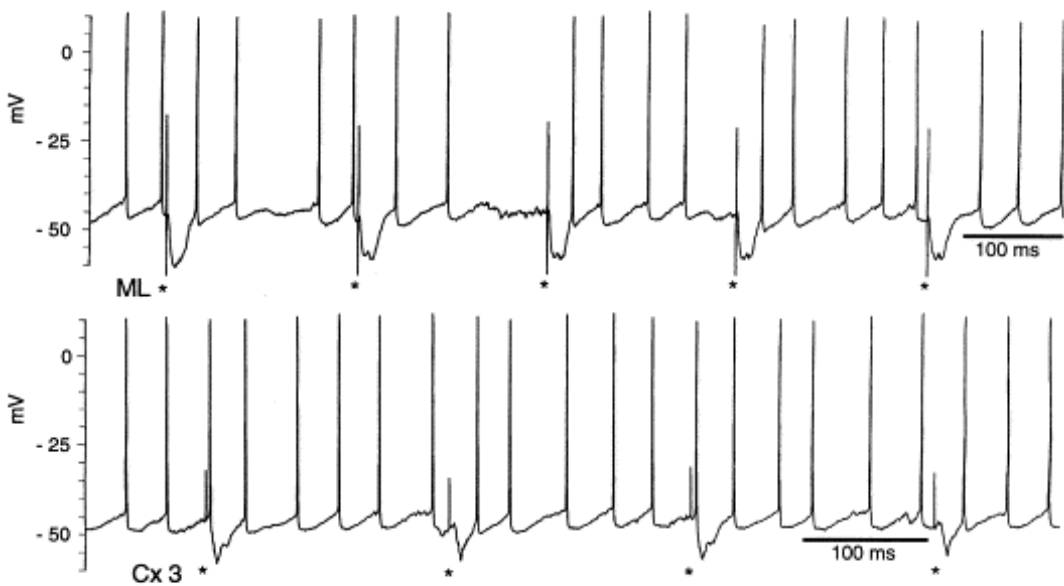

B

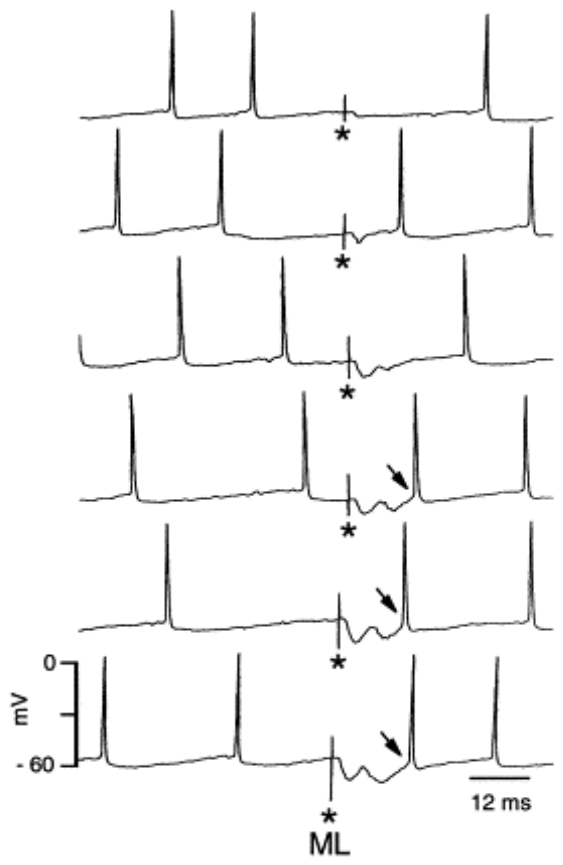

Fig. 4. Recurrent inhibition. (A, B) Same nCL cell. ML and sensorymotor cortex (Cx3) stimulation (A) induced IPSPs. Increasing the ML stimulation intensity (B, from first to last trace) induced compound IPSPs. Stimulus artifacts are marked by asterisks. The arrows point to hyperpolarization-rebound spikes. 


\subsection{Transcortical excitatory responses}

2.4.1. Cuneolemniscal cells. ML stimulation generated presumed transcortical excitatory responses in a total of 16/55 CL neurons (15 bursting, one single spiking). The mean latency of these effects was $8.5 \mathrm{~ms}$ (range 5.5-12 ms) and $3.5 \mathrm{~ms}$ (range 2-7 ms) to ML and cortical stimulation, respectively. This gives a mean latency difference of $5 \mathrm{~ms}$, which is supposedly employed by the lemniscal volley to activate corticocuneate cells. Most of these neurons (11/16) also showed short-latency recurrent responses at similar ML stimulating strengths; no attempt was made to recurrently fire the remaining three cells by moving the ML stimulating electrode. Two examples are shown in Fig. 5 and Fig. 6. Figure 5 illustrates a CL cell (same as Fig. 1A) that also presented excitatory recurrent responses. The cortical stimulus induced a mixed effect with a rapid excitation leading to bursting activity and a post-burst hyperpolarization. The number of spikes within each burst decreased with decreasing stimulating intensity (Fig. 5A; second row in Fig. 5B, and Fig. 5C2). The post-burst hyperpolarization was a synaptic event and not due to intrinsic membrane properties since subthreshold cortical stimulation induced the same sequence of excitation-inhibition (Fig. 5C3) which was not seen when the cell discharged spontaneously (SP in Fig. 5A and 5C1; see also Fig. 1A). Since the ML volleys are expected to activate corticocuneate neurons through the thalamic VPL nucleus, an increase in ML stimulating strength should induce a corticocuneate reflex response. This is shown in the upper row of Fig. 5B where the doublet of spikes is followed by a hyperpolarization and a late depolarization constituted by summed EPSPs. The excitatory and inhibitory late effects were probably produced through different cortical routes since stimulation at different sites in the sensorimotor cortex generated distinct responses (e.g., Fig. 9B). The inset in Fig. 5B shows the superimposition of the late depolarization induced by ML stimulation with the depolarization generated by SSCx stimulation, illustrating the similarity in their time courses. The example in Fig. 6A shows a CL cell that discharged at rest in bursts of three to five spikes separated by neuronal silences of 250-500 ms (showed $\delta$ rhythmicity). ML stimulation induced antidromic responses followed by bursting discharges composed for a full action potential and five to seven subsequent incomplete spikes (panel A1, middle trace). Forelimb motor cortex $[\mathrm{Cx}(3)]$ and forelimb somatosensory cortex $[\mathrm{Cx}(4)]$ stimulation generated basically the same synaptic responses as did ML stimulation except that a full spike at the burst onset was absent (Fig. 6A2). Given the similarity of the late ML and the cortical responses and since the mean burst latencies to ML and cortical stimulation were $6.5 \mathrm{~ms}$ and 3 $\mathrm{ms}$, respectively, the lemniscal volley might have been induced through a transcortical pathway. This was confirmed by data demonstrating that sensorimotor cortical inactivation suppressed the late effects while leaving the antidromic and the recurrent responses (Fig. 6B2). The CL neuron illustrated in Fig. 6B [antidromic identification at just ML suprathreshold intensity (T) in Fig. 6B1] responded to suprathreshold ML stimulating intensity (2T) generating recurrent and transcortical spikes following the antidromic response (Fig. 6B2, upper panel). That the late spikes were transcortically induced was demonstrated in a total of four CL cells that could be recorded before and after cortical inactivation. Inactivation of the sensorimotor cortex by topical application of muscimol eliminated the transcortical responses leaving the antidromic and recurrent spikes (Fig. 6B, lower records). The efficacy of the muscimol-induced inhibition was ascertained by the reduction in the ECoG activity. The amplitude of the ECoG responses to lemniscal stimulation averaged $1.5 \mathrm{mV}$ with the cortex fully active while after muscimol the average amplitude decreased to about $2 \mu \mathrm{V}$ (three animals), probably reflecting thalamic activity. Reversible effects were not seen since the muscimol-induced inactivation lasted for hours. In summary, ML volleys elicited transcortical excitatory responses on CL cells potentiating their burst firing. 


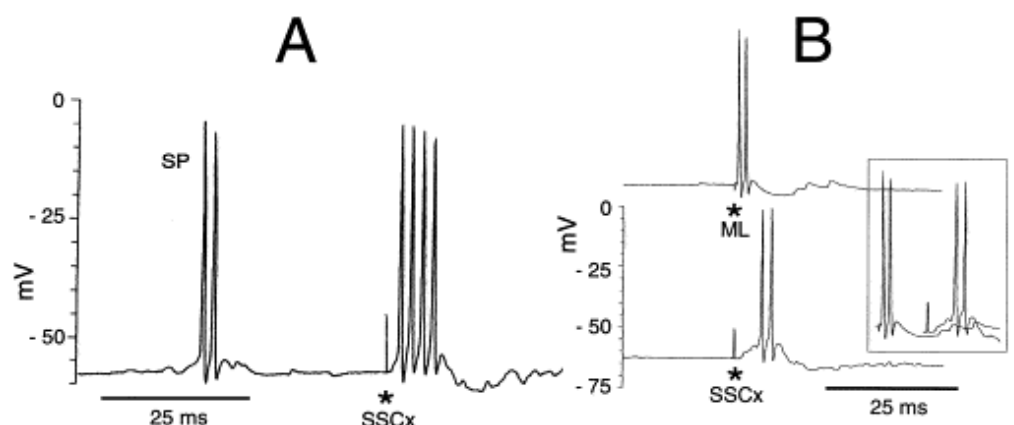

1

C
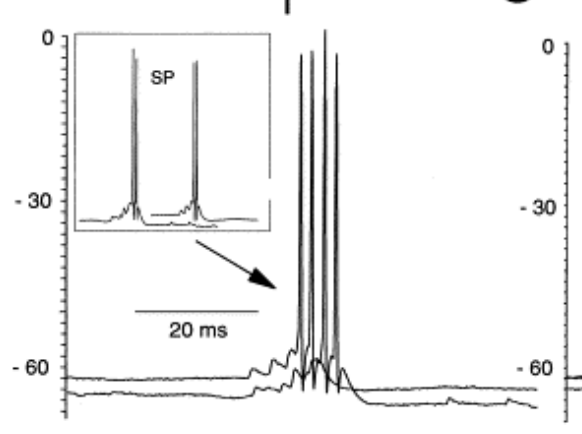

2

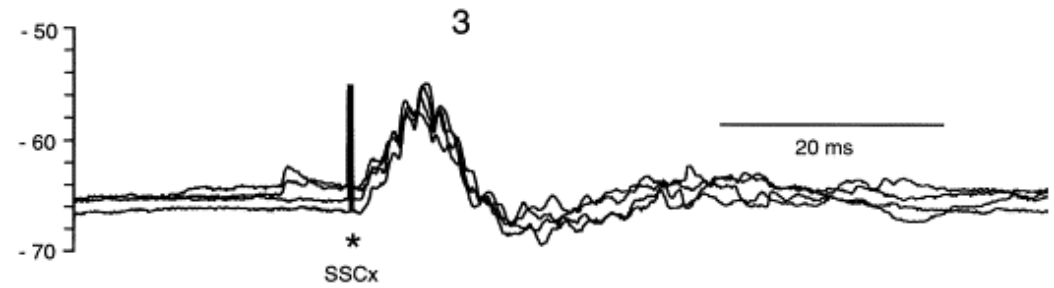

Fig. 5. Transcortical excitation-inhibition. Suprathreshold somatosensory cortex (SSCx) stimulation evoked a burst of four spikes followed by a post-burst hyperpolarization (A; SP = spontaneous) on a CL neuron. ML stimulation generated a late transcortical excitatory response (B, upper record) that had a similar time course to the $\mathrm{SsCx}$ response (B, lower record. Both responses superimposed in the inset). The post-burst hyperpolarization was not spike dependent since it was absent after the spontaneous burst (C1, the traces shown in the inset are superimposed below), but was evoked by just suprathreshold (C2, two superimposed traces) and subthreshold (C3, four superimposed traces) SSCx stimulation. Stimulus artifacts are marked by asterisks. 
B

A

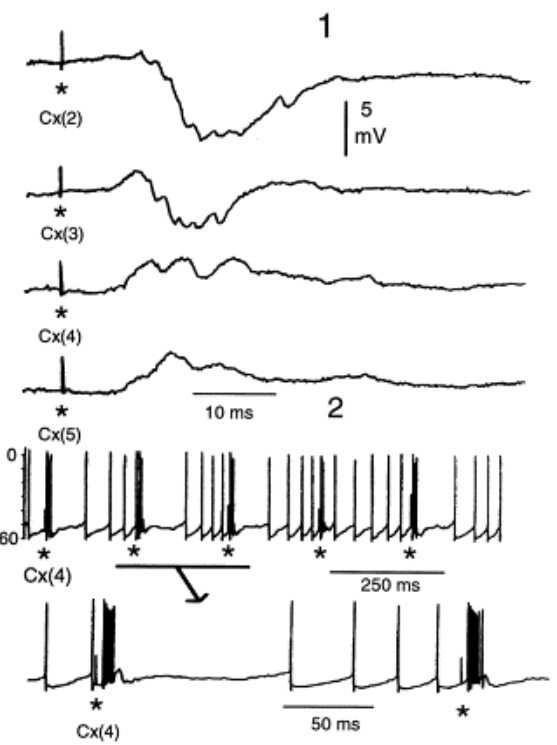

Fig. 9. Synaptic effects induced by sensorimotor cortex stimulation on cuneate neurons. (A) Cuneolemniscal cell generating excitatory responses to somatosensory cortical $[\mathrm{Cx}(4)]$ stimulation. Note decreasing latency with increasing stimulating strength from first to last row. (B) Cuneate non-lemniscal neurons. The responses shown in B1 were obtained by stimulating the intermediate $[\mathrm{Cx}(2)]$ and lateral $[\mathrm{Cx}(3)]$ precruciate motor cortex, and the lateral $[\mathrm{Cx}(4)]$ and intermediate $[\mathrm{Cx}(5)]$ somatosensory cortex, respectively. A different neuron (B2) responded to $\mathrm{Cx}(4)$ stimulation generating bursting activity followed by a slow depolarization with firing arrest. Stimulus artifacts are marked by asterisks.

2.4.2. Non-cuneolemniscal cells. The eight nCL cells that showed transcortical excitatory responses were also excited via recurrent collaterals (Fig. 2A). The cell illustrated in Fig. 2 exemplifies this behavior. The late excitatory responses to ML stimulation (Fig. 2A) were probably due to a transcortical excitation since cortical stimulation induced the same excitatory-inhibitory sequences except that the lemniscal recurrent effects were absent (Fig. 2B). Suprathreshold ML stimulation at $10 \mathrm{~Hz}$ suppressed the transcortical response in most cases, including the post-burst hyperpolarization (lower sweep in Fig. 2A). Sensorimotor cortex stimulation at 10-25 Hz also suppressed the late hyperpolarization (Fig. 2B). Since the latency of the ML transcortical excitatory effect was about $10 \mathrm{~ms}$ and the latency of the directly elicited cortical excitatory response varied from 3 to $6.5 \mathrm{~ms}$, depending on the stimulated site, and given the similarity of both effects, the late EPSP-IPSP sequences induced by ML stimulation were probably induced through the cerebral cortex.

\subsection{Transcortical inhibitory responses}

2.5.1. Cuneolemniscal cells. ML stimulation induced IPSPs on 12/55 CL cells at a mean latency of $11.5 \pm 2.5 \mathrm{~ms}$. This late inhibition is interpreted as being induced through the cortex because cortical inactivation suppressed it in two out of two cells tested (data not shown). Furthermore, cortical stimulation also evoked inhibition on these cells at a shorter latency, compatible with the ML inhibition being induced via a transcortical route. The example shown in Fig. 7 illustrates a CL cell that did not show spontaneous IPSPs at rest but presented subthreshold and suprathreshold depolarizations leading to single or bursting activity (A). Spontaneous IPSPs were evident when the cell was depolarized by intracellularly injecting $0.2 \mathrm{nA}$ of positive direct current (Fig. 7B). With the cell depolarized, the ML stimuli generated antidromic spikes followed by a late hyperpolarization at a latency of about $15 \mathrm{~ms}$ (superimposed responses in Fig. 7C). The late hyperpolarization generated by ML stimulation can be ascribed to a transcortical route. Figure 8A schematizes the position of six pairs of stimulating bipolar electrodes routinely placed in the sensorimotor cortex. The responses of the cell shown in Fig. 7 to stimulation of five of these sites are illustrated in Fig. 8B. The difference in latency between the ML transsynaptic effect (Fig. 7C) and the pericruciate cortical inhibitory responses $(15-6.5=8.5 \mathrm{~ms})$ leaves enough time for the lemniscal volley to follow a thalamo-cortico-cuneate pathway. Transcortical inhibitory effects were also observed in three single spiking CL neurons (data not shown). 


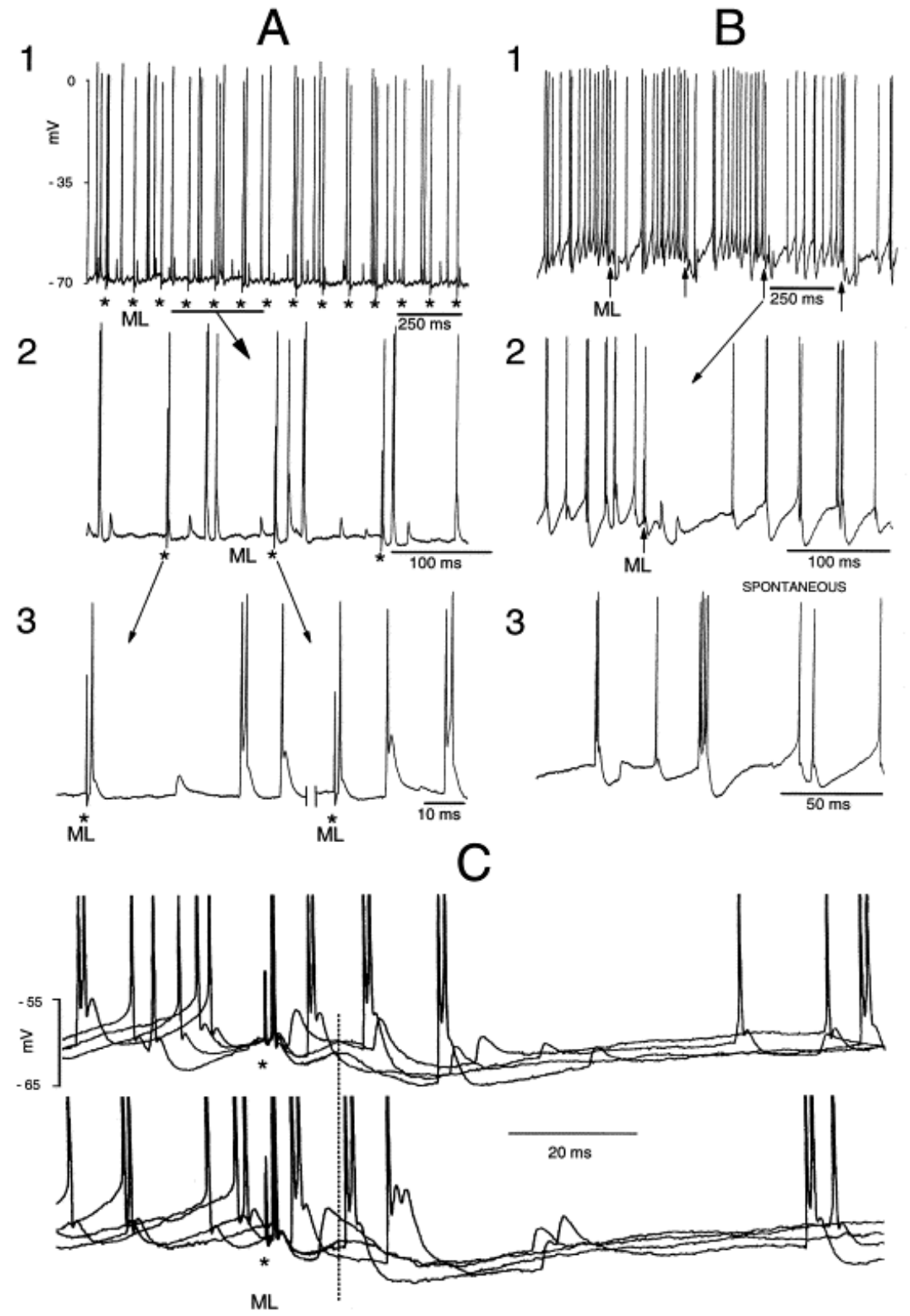

Fig. 7. Transcortical inhibition. ML stimulation induced antidromic responses without apparent postspike effects on a CL cell at rest (A1, the section marked by a horizontal line expanded in A2 with the first two responses to ML stimulation further expanded in A3). With the cell artificially depolarized, ML stimulation evoked transcortical inhibition (B1, one stimulus sequence expanded in B2), and the spontaneous spikes were followed by postspike hyperpolarizations (B3). Sequences of responses to ML stimuli are superimposed in $\mathrm{C}$ (the discontinuous line signals the approximate onset of the late hyperpolarization). Stimulus artifacts are marked by asterisks and arrows. The spikes in $\mathrm{C}$ were truncated.

2.5.2. Non-cuneolemniscal cells. Transcortical inhibitory responses appeared after preceding excitations (Fig. 2A) and were not observed in isolation on nCL neurons.

\subsection{Electrical stimulation of the sensorimotor cortex (Table 1)}

One to $10 \mathrm{~Hz}$ cortical stimulation induced IPSPs and EPSPS on most of the CL and nCL neurons tested, respectively (Table 1). The mean latency to the onset of the IPSPs was $8.5 \mathrm{~ms}$ (range, 7-14 ms) and to the onset of the EPSPs was $5.2 \mathrm{~ms}$ (range, 2.5-8 ms). About 38\% of the CL cells responded to cortical stimulation generating EPSPs (Fig. 9A) or sequences of EPSPs-IPSPs (Fig. 5C3). Sensorimotor cortical stimulation also induced IPSPs on about $22 \%$ of the nCL neurons (Fig. 4A, second row), as well as differential responses on a distinct set of six $\mathrm{nCL}$ cells in which primary motor cortex stimulation 
induced IPSPs and primary somatosensory cortex stimulation induced EPSPs (Fig. 9B1). These latter cells showed mixed excitatory-inhibitory responses when stimulating the precruciate cortex lateral to the tip of the cruciate sulcus, with the rising phase of the EPSPs (mean latency $6.5 \mathrm{~ms}$, range 3.2-9.5 ms) preceding the peak of the IPSPs (mean latency $9 \mathrm{~ms}$, range 7.5-12 ms) by a mean of $10.5 \mathrm{~ms}$ (range, 7-20 $\mathrm{ms}$ ). Finally, five out of $25 \mathrm{nCL}$ neurons excited by cortical stimulation generated burst firing followed by a slow rising depolarization with arrest of firing (Figs 9B2, 10C).

In summary, low-frequency stimulation of the sensorimotor cortex induced inhibition on $62 \%$ of the $\mathrm{CL}$ and excitation on $78 \%$ of the $\mathrm{nCL}$ neurons, with the remaining showing the opposite. In addition, some of the $\mathrm{nCL}$ neurons $(n=6)$ generated short-latency EPSPs to stimulation of the somatosensory cortex and longer-latency IPSPs to stimulation of the precruciate motor cortex.

\subsection{Cortical synchronization makes accessible previously unavailable medial lemniscus-thalamo-cortico- cuneate routes}

Only eight out of 29 of nCL neurons tested were recurrently excited, but since sensorimotor cortex stimulation activates the majority of the $\mathrm{nCL}$ cells, the excitation observed on these neurons by ML stimulation may be considered to be mostly exerted through the cortex. However, four nCL single spiking neurons activated by cortical stimulation could not be demonstrated to respond to ML stimulation. Since the ML volleys are expected to reach the cortex, the assumption is that the intracortical circuitry selectively filters some of the incoming lemniscal signals. To test this hypothesis, paroxysmal activity was electrically induced in the sensorimotor corte ${ }^{37}$ in an attempt to disable the cortical inhibitory mechanisms and thus making transcortically responsive the $\mathrm{nCL}$ neurons that did not respond to ML volleys in control conditions. All four nCL neurons satisfied this assumption.

The example illustrated in Fig. 10 shows that before inducing cortical epileptiform activity, the cell did not respond to ML stimulation (Fig. 10A). Ten to $15 \mathrm{~s}$ after evoking paroxysmal activity, however, the same intensity of stimulation applied to the ML induced both a clear increase in the electrocorticographic activity and a response in the nCL cell (Fig. 10B1). The ML volleys induced the apparition of spikelets and low amplitude spikes as did the cortical paroxysmal "spike" (SP) that also generated barrages of EPSPs. When stimulating the ML at different intervals from the paroxysmal "spike", a refractory period of about $70 \mathrm{~ms}$ for both the cortical gross activity and the intracellular cuneate activity was found (Fig. 10B2-4). During the cortical paroxysm, the cuneate cells remained partially depolarized and showed bursting discharges tightly coupled to the paroxysmal cortical "spike", particularly at the end of the paroxysm. At rest, stimulation of the SSCx (site 4 in Fig. 8) induced bursting activity at a latency of about $4 \mathrm{~ms}$ followed by neuronal silences (Fig. 10C). When the cortical stimulation coincided with the cortical paroxysmal activity the cell still induced bursting responses but the neuronal silences were reduced (Fig. 10D). In addition, the ML transcortical responses exerted on CL neurons were greatly increased following the cortical paroxysms. The example illustrated in Fig. 10E shows a CL cell (collision in the fourth row of Fig. 10E1) transcortically responding to ML stimulation with a doublet of spikes before the cortical paroxysm (Fig. 10E1). However, $12 \mathrm{~s}$ after a cortical paroxysm ML stimulation elicited an increased ECoG activity which supposedly potentiated the burst firing of the CL cell (Fig. 10E2). 
A

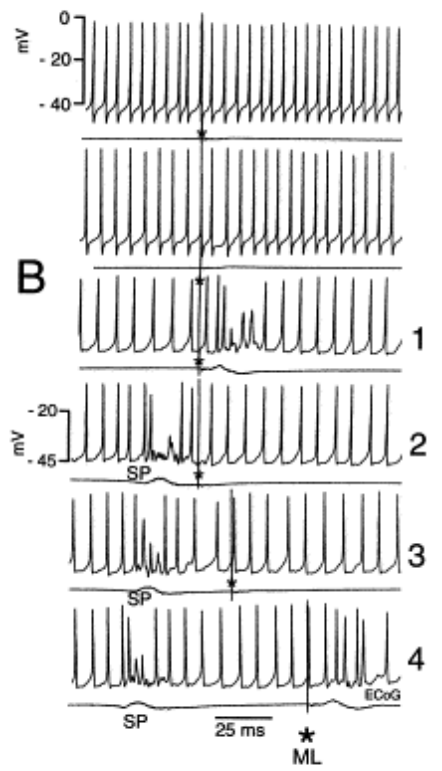

1

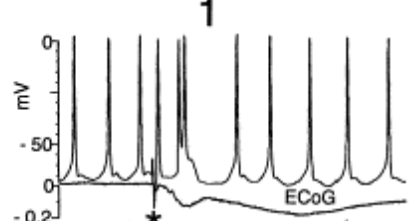

$-0.2$
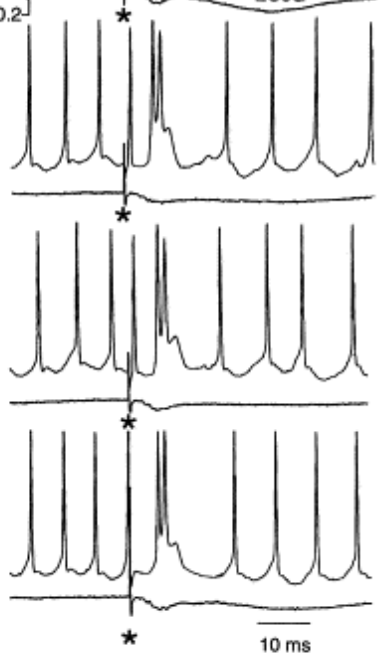

ML
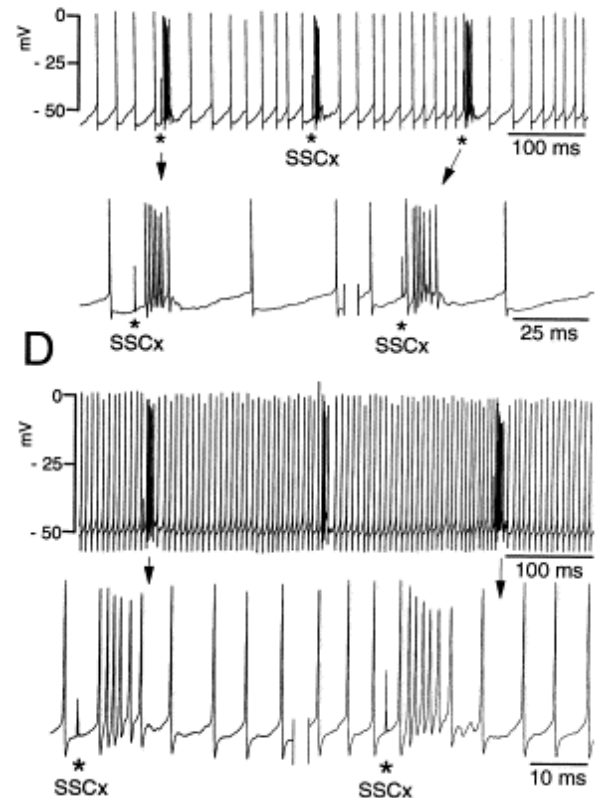

E

2

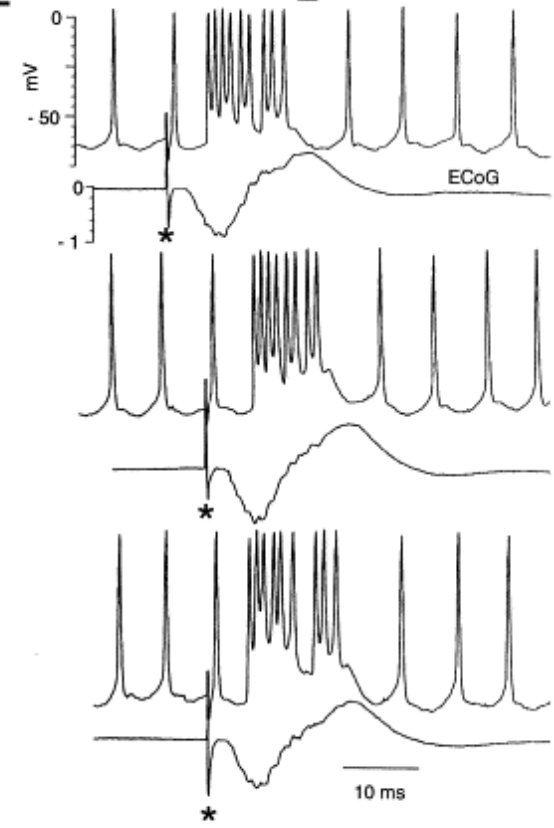

$\mathrm{ML}$

Fig. 10. Cortical synchronization unmasked lemniscal-cortico-cuneate pathways. (A-D) A nCL neuron not responding to ML stimulation (A) but that displayed transcortical responses after paroxysmal cortical activity was induced by a train of electrical stimuli at $100 \mathrm{~Hz}$ for $2 \mathrm{~s}$. (B, SP = spontaneous paroxysmal activity). The same cell responded to somatosensory cortex (SSCx) stimulation before (C) and after (D) the cortical synchronization, with the silent periods following the cortically induced bursts reduced upon membrane depolarization (D). The arrows in C and D signal the expanded portions. (E) A CL cell responding to ML volleys before (E1) and $12 \mathrm{~s}$ after (E2) a cortical paroxysm was electrically induced in the contralateral sensorimotor cortex. Stimulus artifacts are marked by asterisks.

Thus, although every lemniscal volley appears to reach the cortex, the appearance of a cortico-cuneate reflex response as well as its magnitude is determined by the state of the intracortical circuitry. The cortical synchronization appears to disable the intracortical inhibitory processes, allowing some of the lemniscal volleys to follow an otherwise unavailable transcortical route (Fig. 10A,B) and potentiating the responses observed in control conditions (Fig. 10E). 


\subsection{Bursting activity}

The tendency of the cuneate neurons to generate bursting activity can be explained by their intrinsic properties, ${ }^{13 .,}$ 37. and 40. by the activation of lemniscal recurrent collaterals entering the nucleus (Fig. 1), and by reflex activation of the cortico-cuneate loop (Figs 2B, 5, 6). An additional mechanism is constituted by spontaneous EPSPs crossing threshold as demonstrated by the records shown in Fig. 11. The selected records superimposed in Fig. 11D had roughly the same duration, indicating that the EPSPs leading to single and bursting activity varied solely in amplitude. The superimposed records of Fig. 11E resemble the spontaneous bursts of Fig. 11C, indicating that the increased number of spikes shown by a given burst was due to the summation of different EPSPs. If this interpretation is correct, then membrane hyperpolarization should unmask the EPSPs. Since d.c. hyperpolarizing current induced oscillatory activity which led to cellular deterioration, hyperpolarizing pulses of $35 \mathrm{~ms}$ duration and of $0.2-1.5 \mathrm{nA}$ intensity were applied at $1 \mathrm{~Hz}$ in $0.1 \mathrm{nA}$ steps through the recording electrode until observing subthreshold responses (Fig. 12, panel 3). Thus, EPSPs of unknown origin, probably induced by primary afferents, can generate bursting activity on CL cells and interneurons. The bursting activity initiated by giant EPSPs is further potentiated by focused ML recurrent collaterals (Fig. 1 and Fig. 6B) and through the corticocuneate loop (Fig. 2, Fig. 5 and Fig. 6).

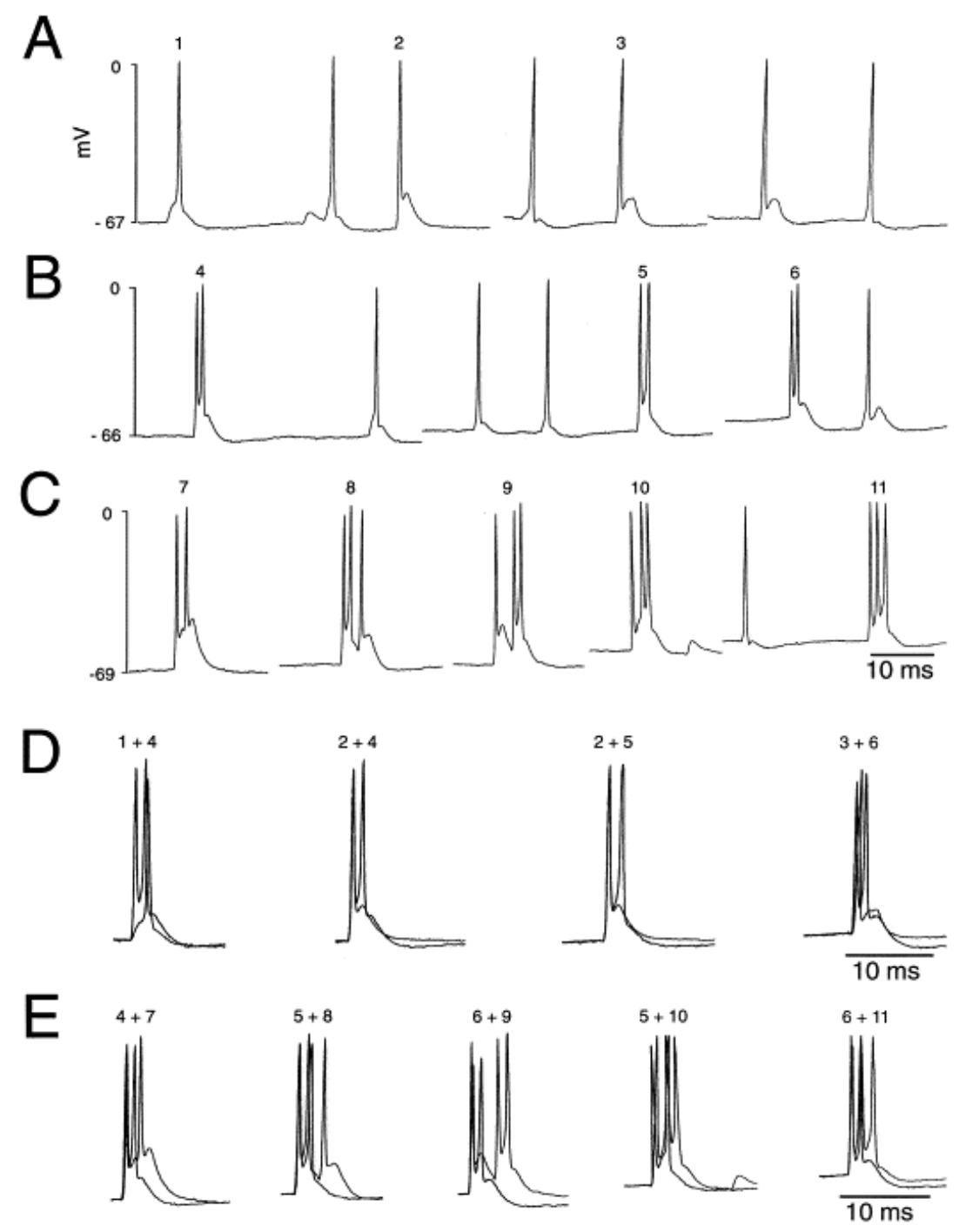

Fig. 11. EPSP-generated spikes. A CL neuron showing spontaneous single spikes (A), doublets (B) and triplets (C). Some samples are superimposed in D and $\mathrm{E}$ as indicated by the numerals at the top. The EPSPs leading to single spikes and doublets varied only in amplitude. The triplets originated by the summation of two EPSPs. 

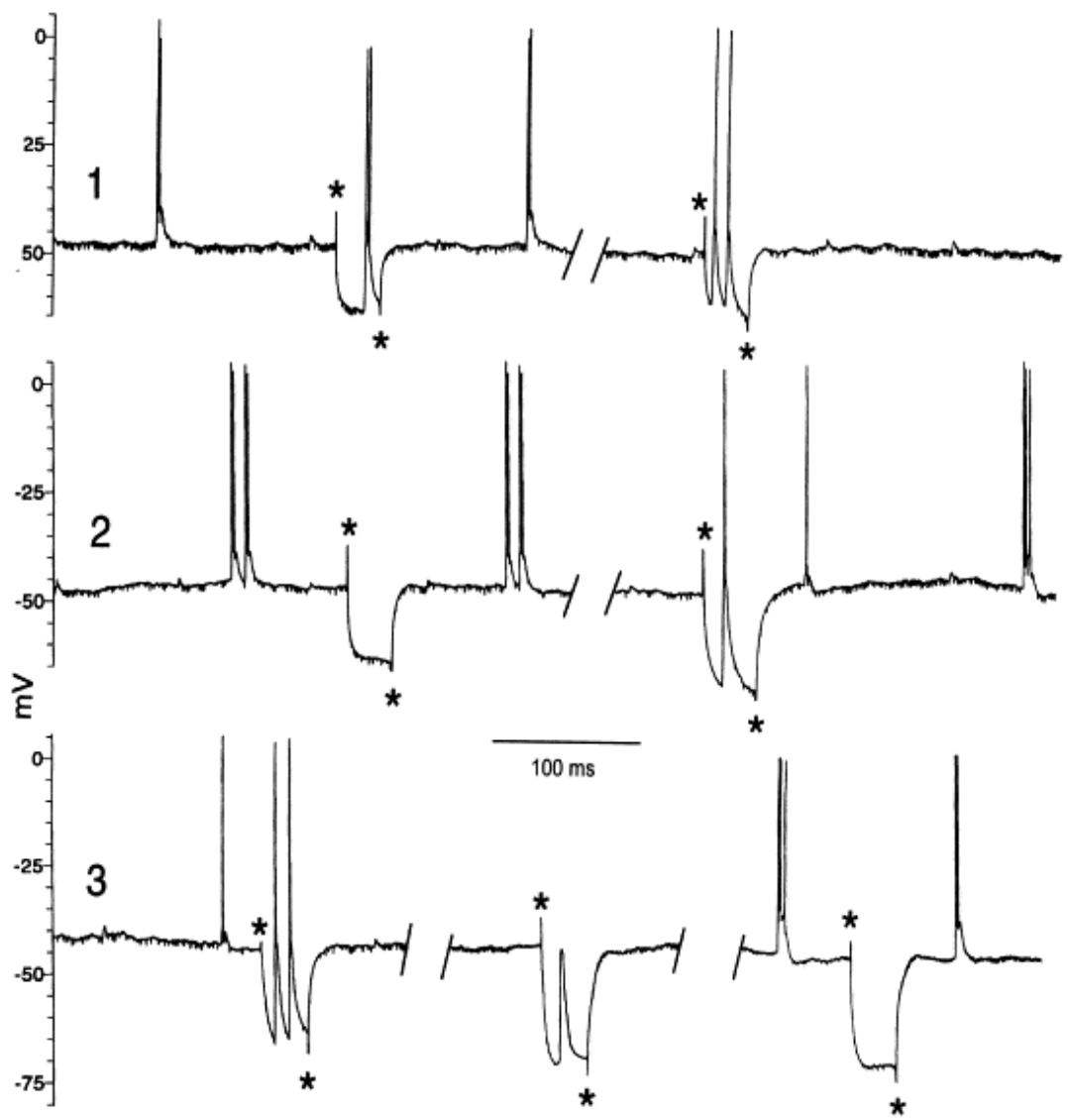

Fig. 12. EPSP-generated spikes. Hyperpolarizing pulses of increasing intensity (panels 1-3) were applied to a CL neuron (the beginning and end of the hyperpolarizing pulses are marked by asterisks) while spontaneously discharging, to uncover the underlying EPSPs (panel 3).

\section{Discussion}

The study revealed four major findings.

1. Medial lemniscal stimulation induced recurrent excitatory and inhibitory effects on both CL and nCL neurons.

2. Sensorimotor cortical stimulation primarily activated nCL cells and inhibited CL neurons, but a substantial proportion of both classes of cells showed the opposite.

3. Impairment of the intracortical circuitry by induction of paroxysmal discharges made available previously inaccessible lemniscal-thalamo-cortico-cuneate routes.

4. Several mechanisms induced repetitive activity in cuneate cells including EPSPs, recurrent collaterals and cortical reflex responses.

\subsection{Recurrent effects}

The presence of presumed recurrent EPSPs and IPSPs appears well documented by the sample records shown in Fig. 1, Fig. 2, Fig. 3, Fig. 4 and Fig. 6B. Amassian and De Vito ${ }^{2}$ were the first to report that ML stimulation induced a repetitive synaptic response of one to two spikes following the antidromic action potentials at a latency of 1.3-3.7 ms. They attributed the late responses to the excitatory synaptic action produced by recurrent collaterals of CL neurons on other CL neurons. An alternative explanation would imply descending fibers activated by the spread of current (e.g., to the cerebral peduncle), but the late spikes were induced at the same or even lower thresholds than the antidromic responses (Fig. 1; see also Gordon and Jukes ${ }^{25}$ ), which points to recurrent effects as the most likely interpretation. The presumed recurrent responses could also be induced by activating aberrant pyramidal fibers running within the $\mathrm{ML}^{31}$ but Gordon and Jukes ${ }^{25}$ reported recurrent effects in the gracile nucleus by stimulating in the 
ventrobasal thalamus, where such fibers are not known, and after removing large parts of the frontal cortex, thus eliminating the possibility of involving a transcortical route (see also Fig. 6B).

Previous work described less incidence of recurrent responses on the cuneate ${ }^{6}$ and the gracile ${ }^{25}$ nuclei in barbiturate anesthetized cats than reported in this study. Since these reports were based on extracellular recordings, subthreshold effects were missing. Gordon and Jukes ${ }^{25}$ kept the ML stimulation intensity below threshold for antidromic activation, which would also account for the low incidence of observations.

The recurrent EPSPs and IPSPs were induced at similar thresholds but the latency of the IPSPS exceeded that of the EPSPs by a mean of $0.7 \mathrm{~ms}$, indicating that an additional neuron might be intercalated in the inhibitory pathway. Since the nCL neurons are mostly, if not exclusively, inhibitory, ${ }^{34 .,}$ 35., 36., 42. and 54. their recurrent activation (Fig. 2) and inhibition (Fig. 3 and Fig. 4) will induce inhibition and disinhibition on the CL cells upon which they synapse, respectively (Fig. 13).

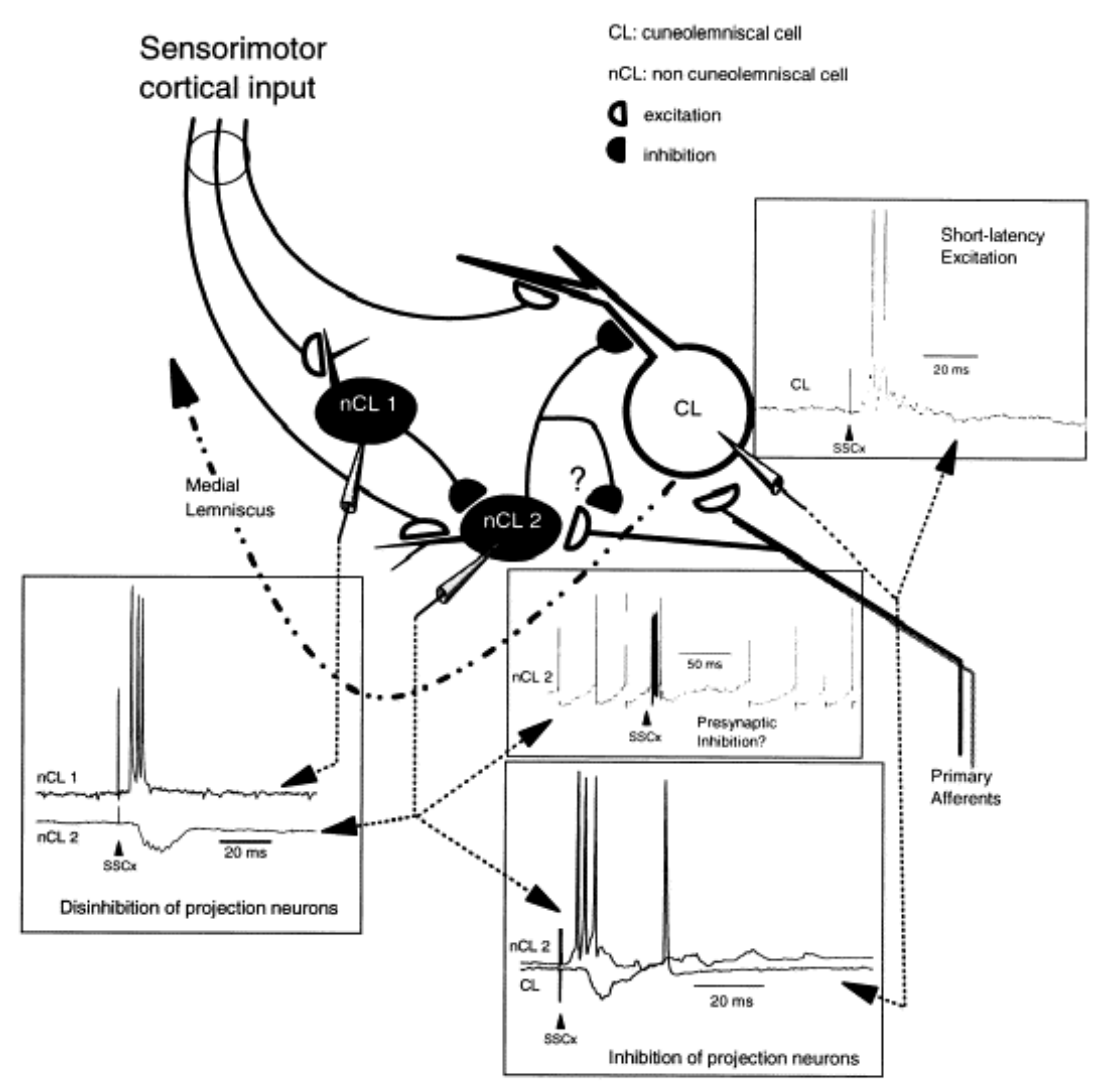

CUNEATE NUCLEUS

Fig. 13. Proposed intracuneate circuitry. It is assumed that the nCL cells are local inhibitory neurons making synaptic contact between them and with CL cells, thus inducing disinhibition and inhibition on projection cells, respectively. The observed corticofugally induced excitatory effects presented a shorter latency than the corticofugally induced inhibitory responses for both classes of neurons (nCL and CL). Accordingly, the pairs of records shown in two of the insets were aligned supposing that the inhibition was produced through an intercalated interneuron (nCL1 or nCL2). The stimulus artifacts to somatosensory cortex (SSCx) stimulation are signaled by arrowheads.

A mechanism enhancing the synchronized firing among functionally related CL neurons may involve positive feedback by recurrent collaterals. The recurrent excitation of CL cells by other CL neurons might partially explain the tendency of these cells to fire in bursts following an afferent volley, since the collaterals are short and supply synaptic boutons to the clusters region. ${ }^{22}$ The functional significance of the recurrent excitation probably consists of inducing bursting activity among closely spaced CL neurons receiving common peripheral input and, thus, tending to synchronize their activity to increment their postsynaptic impact. Consequently, a given CL neuron activated by stimulation of its receptive field would tend to impose its pattern of activity on neighboring cells. This is possible because the CL neurons are somatotopically organized so that neurons processing a single cutaneous submodality in the middle 
region of the cuneate are adjacent to each other. ${ }^{19}$ Therefore, neighboring CL neurons with overlapping receptive fields would receive excitatory input from fibers ascending in the dorsal columns and from recurrent collateral branches from axons of neighboring CL cells, generating bursting and synchronous activity.

The ML volleys induced recurrent excitation followed by a late transcortical excitation on eight $\mathrm{nCL}$ cells (Fig. 2). The functional role of these interactions may shape the peripheral receptive fields of cuneate neurons by disinhibiting the transmission through strongly activated CL cells while inhibiting the rest. These putative effects need to be very focused since the VPL thalamocortical neurons with a given receptive field cluster together and closely reflect the receptive fields of cells in the dorsal column nuclei. $^{49}$

Increasing the intensity of ML stimulation revealed two components in the recurrent inhibition exerted on nCL neurons, an early and a late IPSP (Fig. 4). Assuming that both responses were generated through an intercalated inhibitory cell, they can be ascribed to different mechanisms, such as distinct pathways, whether intranuclear or extranuclear (e.g., through the bulbar reticular formation), different postsynaptic receptors (e.g., GABAa and GABAb), to the activation of low-threshold and high-threshold recurrent axons (e.g., with distinct diameters or at a different distance from the stimulating electrode), or to an interneuron discharging various spikes producing the first and second components of the IPSP.

\subsection{Transcortical effects}

Orthodromic ML volleys acting on the nucleus through thalamus and cerebral cortex were observed on CL and nCL neurons. The corticocuneate input constitutes a true feedback since the gross discharge evoked in the nucleus by peripheral cutaneous stimulation or by direct stimulation of the dorsal columns is followed by a second late discharge which disappears upon removal of the cortex. ${ }^{51}$ The transcortical effects observed on CL neurons were excitatory (Fig. 6 and Fig. 10), inhibitory (Fig. 7) or showed mixed effects with excitation preceding inhibition (Fig. 5). Ultrastructural results using anterograde degeneration methods ${ }^{53}$ and electron microscopical observations ${ }^{14}$ revealed that the corticofugal terminals contact fine dendrites of cuneate neurons. This may be the morphological basis that could explain the appearance of the cortically induced spikelets in some CL neurons (Fig. 6A). The spikelets were probably generated in the dendrites and transmitted electrotonically or actively to the soma, where they could eventually trigger a full response. Less probably, they could also be a transmembrane reflection of the neuronal activity in the neighborhood of the impaled cell or induced through electrical synapses, although gap junctions have not been reported in the cuneate (but see Ref. 40). Alternatively, they might represent a spike block.

The axons of neighboring CL neurons synapse with adjacent thalamocortical cells which, in turn, activate clusters of corticocuneate neurons in the sensorimotor cortex. The role of the cortex would be to enhance the activity of CL neurons strongly activated from the periphery and, through intercalated nCL cells, either inhibit specific ensembles of surrounding CL cells or promote a general inhibition on all CL neurons. In the first case, the cortex would induce a central zone of activity surrounded by a peripheral zone of inhibition. In the second, it would produce a general inhibition on the cuneothalamic transmission so that only the CL neurons able to overcome the underlying inhibition would transmit to the thalamus. In both cases the neocortex will be able to discriminate wanted from unwanted sensory information at the level of the DCN.

The descending corticocuneate transmission appears to be intracortically selected since the ML volleys that did not induce (Fig. 10A) or did elicit secondary responses (Fig. 10E1) in control conditions affected the cuneate (Fig. 10B) and potentiated the secondary responses (Fig. 10E2), respectively, when the intracortical circuitry was disrupted by inducing paroxysmal cortical activity. This could indicate that the intracortical inhibitory mechanisms would specifically select, in physiological conditions, the most appropriated transcortical route according to functional requirements. The high degree of divergence of the thalamocortical cells ${ }^{44}$ allows for different intracortical pathways, some of which may be tonically inhibited and unmasked under specific circumstances. In the non-anesthetized animal, these different pathways could be selected according to the most relevant sensory information to accomplish purposive sensorimotor activities as, for example, active exploration and manipulation of objects. As illustrated by the data shown in Fig. 1, Fig. 2 and Fig. 6, the transcortical effects would tend to potentiate the recurrent responses. 


\subsection{Synaptic responses induced by cortical stimulation}

Single stimuli applied to the sensorimotor cortex, at 1-10 Hz, induced EPSPs or sequences of EPSPsIPSPs on $38 \%$ of the CL neurons (Fig. 5), and IPSPs on $22 \%$ of the nCL cells (Fig. 4). That stimulation of the cerebral cortex mostly excites DCN cells projecting in the medial lemniscus and inhibits nCL neurons has been amply reported using intracellular ${ }^{46}$ and extracellular ${ }^{16 .,}$ 23., 24., 25., 33. and 41. techniques. The present data corroborate the results of Schwartzkroin et al., ${ }^{46}$ but differ from other intracellular studies ${ }^{7}$ and 13. reporting no evidence for postsynaptic excitation of CL cells or postsynaptic inhibition of nCL cells to direct cortical stimulation. In this study, the stimulating technique was improved by placing a groundisolated constant current unit as close to the preparation as possible to reduce the shock artifacts. This proved to be important, for the majority of the excitatory effects had small latencies that will otherwise be "artifacted" and thus pass undetected. Stimulation was applied at a gradual increasing intensity within the primary motor and primary sensory cortexes, while in our previous study, ${ }^{13}$ only the primary motor cortex was stimulated at supramaximal intensity which could obliterate the excitatory effects. The cortical-induced excitatory effects were the fastest, which probably indicates that they were produced through collateral branches of corticospinal cells since the corticocuneate axons from non-corticospinal neurons are slow-conducting. ${ }^{38}$ The observation of cortically evoked excitatory and inhibitory influences on CL neurons suggests a duality in the cortical modulation exerted on the ascending somesthetic information: amplification of selected messages with simultaneous inhibition of the unwanted ones.

The bursting discharges induced by cortical stimulation on some nCL cells were followed by silenced firing coincident with a slow rising depolarization (Figs 9B2, 10C). If the resting tonic firing showed by these cells was due to primary afferent activity, the cortically induced bursts might induce presynaptic depolarization of the afferent terminals leading to presynaptic inhibition. ${ }^{5}$ and 7 .

Some nCL neurons were reciprocally affected by SI and MI stimuli (Fig. 9B1). Therefore, the putative disinhibition exerted by MI cuneolemniscal transmission will be annulled by the inhibition induced by SI if both processes occur simultaneously. The functional significance of such an interaction is difficult to explain. One possibility is that the motor strategy selected in MI would predict the peripheral receptors to be activated by the movement itself to disinhibit the appropriate CL cells in advance. When activated, these same CL neurons would generate a negative feedback inhibiting themselves through SI. Such a mechanism could serve to differentially filter the signals not able to overcome the underlying inhibition while allowing others more strongly activated to be transmitted to the thalamus, helping to make the receptive fields smaller and more discrete as, for example, during active touch.

\subsection{Bursting activity}

The bursting activity normally seen in cuneate neurons appears to be due to several mechanisms including intrinsic membrane properties, ${ }^{13 .,}$ 37. and ${ }^{40}$ recurrent collaterals (Fig. 1), cortical reflex responses (Figs 2, 5, 6), and EPSP-generated spikes (Fig. 11 and Fig. 12).

That many of the excitations leading to bursting spikes in cuneate neurons were EPSPs and not excitatory rebounds due to activated cationic currents at subthreshold membrane potentials ${ }^{37 . \text { and }} 40$. is demonstrated by the data shown in Fig. 11 and Fig. 12. Repetitive activity can be generated by EPSPs crossing threshold during sufficient time to induce various spikes. This is possible since somatic afferent fibers exert a powerful monosynaptic excitation on CL neurons that can be extracellularly seen as unitary prepotentials preceding the spikes. ${ }^{3}$ In addition, the EPSPs induced by stimulating primary afferent fibers have been shown to be unusually large and to elicit bursting activity. ${ }^{7}$ These synaptic contacts are so powerful that a single primary afferent fiber can generate doublets or triplets of output spikes from several target neurons ${ }^{20}$ without the need for temporal or spatial summation. ${ }^{28}$ The morphological basis that explain this strong synaptic influence is large synaptic knobs ${ }^{45}$ in the terminals of dorsal column afferent glutamatergic fibers ${ }^{17}$ synapsing on CL cells. The high variability in the amplitude of the EPSPs (Fig. 11) might be due to the fact that the primary afferents make not only terminal but also en passant synaptic contacts with cuneate neurons. ${ }^{21}$

\subsection{Cuneate circuitry (Fig. 13)}

Some conclusions regarding corticocuneate and intracuneate interactions can be made from the present and a previous report. ${ }^{37}$ First, the more direct corticocuneate effect is excitation since corticofugally evoked excitation always occurred at a shorter latency than the corticofugally evoked inhibition. Secondly, inhibition induced by cortical stimulation is the first effect in the majority of CL neurons and in a substantial proportion of nCL cells (Table 1). The corticofugal inhibition of inhibitory 
nCL cells synapsing with CL neurons allows for corticofugally induced disinhibition of CL cells. Disinhibition of CL cells can also be induced by stimulation of the dorsal columns. ${ }^{11}$ Thirdly, the corticofugal induction of burst firing in nCL neurons would induce a strong inhibition on CL cells which could be terminated by presynaptic inhibition of the activated nCL neurons through axons from these same nCL neurons or via a different pool of corticofugally activated nCL cells. ${ }^{6}$ Lastly, the ML recurrent effects on cuneate cells were usually potentiated through the cerebral cortex (Fig. 1, Fig. 2 and Fig. 6; see also Fig. 11 in Canedo $^{11}$ ).

The circuit design depicted in Fig. 13 (the collaterals have been omitted for clarity) also explains recent work indicating that the dorsal column-medial lemniscal system may be particularly important for frequency and duration discrimination of tactile information. ${ }^{52}$ Thus, the cortico-DCN modulation might contribute to the selection of certain inputs to the detriment of others as well as to the adequate frequency and duration discrimination for reliable transmission of somesthetic information. Focused cortical activation and removal of inhibition would guarantee the adequate transmission of high-frequency inputs.

\section{Conclusions}

In the alert animal, the corticocuneate fibers may serve to increase the gain of the input for featurelinked events detected by the cerebral cortex. The corticocuneate feedback loop may select input signals according to the demands of neocortical processing, as in the visual pathway. ${ }^{48}$ The cuneate modulation by corticofugal inputs may be important given the abundance of corticocuneate axons which, together with the corticothalamic feedback, might enable the cerebral cortex to accurately modulate the ascending signals at both levels of processing. During quiet sleep, the cerebral cortex will impose its rhythmical patterns on the cuneate, inducing a state of resonant activity by matching the preferred intrinsic cuneate rhythmicity. ${ }^{37}$ During wakefulness, the cortex will select and process the more relevant somatosensory information by potentiating its pass through the cuneate and the thalamus while inhibiting the rest.

The cuneate neurons accounting for the response selectivity of CL cells are inhibitory neurons located in the cuneate itself and in the neighboring reticular formation, and recurrent collateral branches from CL axons. The interneurons are involved in stimulus-specific inhibitory responses ${ }^{7 \text {. and }} 46$. and also probably contribute to cuneate oscillations. ${ }^{37}$ They are likely to be implicated in discrimination processes that will increase as the corticofugal input to these cells also increases. Thus, a major tactile resolution and manipulative skills in those animal species with an increased corticocuneate input would be expected. This will be owing to an increased corticofugal potentiation of selected inputs and an increased inhibition of other inputs. A link between tactile discrimination and manipulative skills is likely to occur since exploratory behavior links movement skills to discriminative capacity. Thus, it has been reported that the corticospinal fibers branching into the DCN are more numerous in monkeys than in cats, ${ }^{9}$ and that most of these corticospinal branching fibers terminate in the cervical cord, at least in felines, ${ }^{38}$ which points to a double functional role for these cells. They might participate in regulating forelimb movement and in the selection of ascending DCN-thalamo-cortical input.

In conclusion, it is suggested that the DCN are actively implicated in constraining the afferent signals through intranuclear inhibitory processes and that participate in integrative functions leading to input selection and assuring frequency and duration discrimination according to the constraints imposed by the sensorimotor corticofugal demands.

\section{Acknowledgements}

The technical assistance of Mr Francisco García Pablo is greatly appreciated. This work was supported by a grant from the DGICYT (PB96-0958). 


\section{References}

1. R.J. Adkins, R.W. Morse, A.L. Towe. Control of somatosensory input by cerebral cortex. Science, 153 (1966), pp. $1020-1022$

2. V.E. Amassian, J.L. DeVito. La transmission dans le noyau du Burdach (nucleus cuneatus). Etude analytique par unités isolées d'un relais somatosensoriel primaire. Coll. Int. Centre Nat. Rech. Sci., 67 (1957), pp. 353-393

3. V.E. Amassian, D. Giblin. Periodic components in steady-state activity of cuneate neurons and their possible role in sensory coding. J. Physiol., Lond., 243 (1974), pp. 353-385

4. Amassian V. E., Macy J., Waller H. J., Leader H. S. and Swift M. (1964) Transformation of afferent activity at the cuneate nucleus. In Information Processing in the Nervous System (eds Gerard R. W. and Duyff J. W.). Excerpla Medica, Amsterdam.

5. P. Andersen, J.C. Eccles, R.F. Schmidt, T. Yokota. Depolarization of presynaptic fibers in the cuneate nucleus. J. Neurophysiol., 27 (1964), pp. 92-106

6. P. Andersen, J.C. Eccles, R.F. Scmidt, T. Yokota. Identification of relay cells and interneurons in the cuneate nucleus. J. Neurophysiol., 27 (1964), pp. 1080-1095

7. P. Andersen, J.C. Eccles, T. Oshima, R.F. Schmidt. Mechanisms of synaptic transmission in the cuneate nucleus. J. Neurophysiol., 27 (1964), pp. 1096-1116

8. A.I. Basbaum, P.J. Hand. Projections of cervico-thoracic dorsal roots to the cuneate nucleus of the rat with observations on cellular bricks. J. comp. Neurol., 148 (1973), pp. 347-360

9. M. Bentivoglio, A. Rustioni. Corticospinal neurons with branching axons to the drosal column nuclei in the monkey. J. comp. Neurol., 253 (1986), pp. 260-276

10. S.R. Cajal. Histologie du Système Nerveux de l'Home et des Vertébrés, Maloine, Paris (1909)

11. A. Canedo. Primary motor cortex influences on the descending and ascending systems. Prog. Neurobiol., 51 (1997), pp. 287-335

12. A. Canedo, J. Mariño. Corticofugal effects on somatosensory ascendant transmission on the cuneate nucleus of chloralose-anaesthetized cats. J. Physiol., Lond., 509 (1998), pp. 31-32S

13. A. Canedo, L. Martinez, J. Mariño. Tonic and bursting activity in the cuneate nucleus of the chloralose anesthetized cat. Neuroscience, 84 (1998), pp. 603-617

14. S. Cheema, R. Fyffe, A. Light, A. Rustioni. Arborizations of single corticofugal axons in the feline cuneate nucleus stained by iontophoretic injection of horseradish peroxidase. Brain Res., 290 (1984), pp. 158-164

15. J.D. Cole, G. Gordon. Timing of corticofugal actions on gracile and cuneate nuclei of the cat. J. Physiol., Lond., 340 (1983), pp. $139-152$

16. J.D. Cole, G. Gordon. Corticofugal actions on lemniscal neurons of the cuneate, gracile and lateral cervical nuclei of the cat. Expl Brain Res., 90 (1992), pp. 384-392

17. S. De Biasi, A. Rustioni. Ultrastructural immunocytochemical localization of excitatory amino acids in the somatosensory system. J. Histochem. Cytochem., 38 (1990), pp. 1745-1754

18. R.W. Dykes, A.D. Craig. Control of size and excitability of mechanosensory receptive fields in dorsal column nuclei by homolateral dorsal horn neurons. J. Neurophysiol., 80 (1998), pp. 120-129

19. R.W. Dykes, D.D. Rasmusson, D. Sretavan, N.B. Rehman. Submodality segregation and receptive-field sequences in cuneate, gracile, and external cuneate nuclei of the cat. J. Neurophysiol., 47 (1982), pp. 389-416

20. D.G. Ferrington, M.J. Rowe, R.P.C. Tarvin. Actions of single sensory fibers on cat dorsal column nuclei neurons: vibratory signalling in a one-to-one linkage. J. Physiol., Lond., 386 (1987), pp. 293-309

21. R.E.W. Fyffe, S.S. Cheema, A. Rustioni. Intracellular staining study of the feline cuneate nucleus. I. Terminal patterns of primary afferent fibers. J. Neurophysiol., 56 (1986), pp. 1268-1283

22. R.E.W. Fyffe, S.S. Cheema, A.R. Light, A. Rustioni. Intracellular staining study of the feline cuneate nucleus. II. Thalamic projecting neurons .J. Neurophysiol., 56 (1986), pp. 1284-1296

23. G. Gordon, M.G.M. Jukes. Correlation of different excitatory and inhibitory influences on cells in the nucleus gracilis of the cat. Nature, 196 (1962), pp. 1183-1185

24. G. Gordon, M.G.M. Jukes. Dual organization of the exteroceptive components of the cat's gracile nucleus. J. Physiol., Lond., 173 (1964), pp. 263-290

25. G. Gordon, M.G.M. Jukes. Descending influences on the exteroceptive organizations of the cat's gracile nucleus. J. Physiol., Lond., 173 (1964), pp. 291-319

26. G. Gordon, C.H. Paine. Functional organization in the nucleus gracilis of the cat. J. Physiol., Lond., 153 (1960), pp. 331-349

27. G. Gordon, W.A. Seed. An investigation of nucleus gracilis of the cat by antidromic stimulation. J. Physiol., Lond., 155 (1961), pp. 589-601

28. B.D. Gynther, R.M. Vickery, M.J. Rowe. Transmission characteristics for the 1:1 linkage between slowly adapting type II fibers and their cuneate target neurons in the cat. Expl Brain Res., 105 (1995), pp. 67-75

29. F. Harris, S.J. Jabbur, R.W. Morse, A.L. Towe. Influence of the cerebral cortex on the cuneate nucleus of the monkey. Nature, 208 (1965), pp. 1215-1216

30. J.B. Hursh. Relayed impulses in ascending branches of dorsal root fibers. J. Neurophysiol., 3 (1940), pp. 166-174

31. H.G.J.M. Kuypers. An anatomical analysis of cortico-bulbar connexions to the pons and lower brain stem in the cat. J. Anat., 92 (1958), pp. 198-218

32. H.G.J.M. Kuypers, J.D. Tuerk. The distribution of the cortical fibers within the nuclei cuneatus and gracilis in the cat. J. Anat., 98 (1964), pp. 143-162

33. M. Levitt, M. Carreras, C.N. Liu, W.W. Chambers. Pyramidal and extrapyramidal modulation of somatosensory activity in gracile and cuneate nuclei. Archs Ital Biol., 102 (1964), pp. 197-229 
34. J.H. Lue, J.Y. Shieh, C.Y. Wen, K.N. Chen, S.A. Chan. GABAergic boutons establish synaptic contacts with the soma and dendrites of cuneothalamic relay neurons in the rat cuneate nucleus. Expl Brain Res., 98 (1994), pp. $13-20$

35. J.H. Lue, Y.F. Jiangshieh, J.Y. Shieh, E.A. Ling, C.Y. Wen. Multiple inputs of GABA-immunoreactivity neurons in the cuneate nucleus of the rat. Neurosci. Res., 27 (1997), pp. 123-132

36. J.H. Lue, S.M. Lai, T.J. Wang, J.Y. Shieh, C.Y. Wen. Synaptic relationships between corticocuneate terminals and glycine-immunoreactive neurons in the rat cuneate nucleus. Brain Res., 771 (1997), pp. 167-171

37. J. Mariño, A. Canedo, J. Aguilar. Sensorimotor cortical influences on cuneate nucleus rhythmic activity. Neuroscience, 95 (1999), pp. 657-673

38. L. Martinez, J.A. Lamas, A. Canedo. Pyramidal tract and corticospinal neurons with branching axons to the dorsal column nuclei of the cat. Neuroscience, 68 (1995), pp. 195-206

39. J. Millar, A.I. Basbaum. Topography of the projection of the body surface of the cat to cuneate and gracile nuclei. Expl Neurol., 49 (1975), pp. 281-290

40. A. Núñez, W. Buño. In vitro electrophysiological properties of rat dorsal column nuclei neurons. Eur. J. Neurosci., 11 (1999), pp. 1865-1876

41. A. Palmeri, M. Bellomo, R. Giufrida, S. Sapienza. Motor cortex modulation of exteroceptive information at bulbar and thalamic lemniscal relays in the cat. Neuroscience, 88 (1999), pp. 135-150

42. A. Popratiloff, J.G. Valtschanoff, A. Rustioni, R.J. Weinberg. Colocalization of GABA and glycine in the rat dorsal column nuclei. Brain Res., 706 (1996), pp. 308-312

43. B.H. Pubols, J.H. Haring, M.J. Rowinski. Patterns of resting discharge in neurons of the racoon main cuneate nucleus. J. Neurophysiol., 61 (1989), pp. 1131-1141

44. E. Rausell, L. Bickford, P.R. Manger, T.M. Woods, E.G. Jones. Extensive divergence and convergence in the thalamocortical projection to monkey somatosensory cortex. J. Neurosci., 18 (1998), pp. 4216-4232

45. I. Rozsos. The synapses of Burdach's nucleus. Acta Morphol. Acad. Scient. Hung., 8 (1958), pp. 105-108

46. P.A. Schwartzkroin, H. Vand Duijn, D.A. Prince. Effects of projected cortical epileptiform discharges on unit activity in the cat cuneate nucleus. Expl Neurol., 43 (1974), pp. 106-123

47. H.-C. Shin, J.K. Chapin. Mapping the effects of motor cortex stimulation on single neurons in the dorsal column nuclei of the rat: direct responses and afferent modulation. Brain Res. Bull., 22 (1989), pp. 242-252

48. A.M. Sillito, H.E. Jones, G.L. Gerstein, D.C. West. Feature-linked synchronization of thalamic relay cell firing induced by feedback from the visual cortex. Nature, 369 (1994), pp. 479-482

49. Steriade M., Jones E. G. and McCormick D. A. (1997) The relay function of the thalamus during brain activation. In Thalamus (eds Steriade M., Jones E. G. and McCormick D. A.), Vol. I., Chap. VI. Elsevier, Amsterdam.

50. P.O. Therman. Transmission of impulses through the Burdach nucleus. J. Neurophysiol., 4 (1941), pp. 153-166

51. A.L. Towe, I.D. Zimmerman. Peripherally evoked cortical reflex in the cuneate nucleus. Nature, 194 (1962), pp. $1250-1251$

52. C.J. Vierck Jr. Impaired detection of repetitive stimulation following interruption of the dorsal spinal column in primates. Somatosen. Motor Res., 15 (1998), pp. 157-163

53. F. Walberg. Axoaxonic contacts in the cuneate nucleus, probable basis for presynaptic inhibition. Expl Neurol., 13 (1965), pp. 218-231

54. C.Y. Wen, K.N. Chen, J.H. Lue, S.A. Chan, J.Y. Shieh. An electron microscopic and morphometric study on the GABA-immunoreactive terminals in the cuneate nucleus of the rat. J. Anat., 181 (1992), pp. 409-415 